\title{
Two-receiver measurements of phase velocity: cross-validation of ambient-noise and earthquake-based observations
}

\author{
Emanuel D. Kästle, ${ }^{1}$ Riaz Soomro, ${ }^{2}$ Cornelis Weemstra, ${ }^{3}$ Lapo Boschi ${ }^{1}$ \\ and Thomas Meier $^{2}$ \\ ${ }^{1}$ Laboratoire iSTeP, Université Pierre et Marie Curie, Paris, France. E-mail: emanuel.kaestle@etu.upmc.fr \\ ${ }^{2}$ Institute of Geosciences, Kiel, SH, Germany \\ ${ }^{3}$ Department of Geoscience and Engineering, Delft University of Technology, Delft, The Netherlands
}

Accepted 2016 September 19. Received 2016 July 25; in original form 2015 December 15

\begin{abstract}
SUMMAR Y
Phase velocities derived from ambient-noise cross-correlation are compared with phase velocities calculated from cross-correlations of waveform recordings of teleseismic earthquakes whose epicentres are approximately on the station-station great circle. The comparison is conducted both for Rayleigh and Love waves using over 1000 station pairs in central Europe. We describe in detail our signal-processing method which allows for automated processing of large amounts of data. Ambient-noise data are collected in the 5-80 s period range, whereas teleseismic data are available between about 8 and $250 \mathrm{~s}$, resulting in a broad common period range between 8 and $80 \mathrm{~s}$. At intermediate periods around $30 \mathrm{~s}$ and for shorter interstation distances, phase velocities measured from ambient noise are on average between 0.5 per cent and 1.5 per cent lower than those observed via the earthquake-based method. This discrepancy is small compared to typical phase-velocity heterogeneities (10 per cent peak-to-peak or more) observed in this period range. We nevertheless conduct a suite of synthetic tests to evaluate whether known biases in ambient-noise cross-correlation measurements could account for this discrepancy; we specifically evaluate the effects of heterogeneities in source distribution, of azimuthal anisotropy in surface-wave velocity and of the presence of near-field, rather than far-field only, sources of seismic noise. We find that these effects can be quite important comparing individual station pairs. The systematic discrepancy is presumably due to a combination of factors, related to differences in sensitivity of earthquake versus noise data to lateral heterogeneity. The data sets from both methods are used to create some preliminary tomographic maps that are characterized by velocity heterogeneities of similar amplitude and pattern, confirming the overall agreement between the two measurement methods.
\end{abstract}

Key words: Numerical approximations and analysis; Tomography; Interferometry; Surface waves and free oscillations; Wave propagation.

\section{INTRODUCTION}

It has been observed that an important contribution to what is classically considered as noise in a seismogram comes from signals that arise from the oceans' coupling with the solid Earth. These signals are generally referred to as microseisms and their generation has first been explained by Longuet-Higgins (1950) and Hasselmann (1963). Their dependence on the ocean activity is reflected by a seasonal variability of the recorded noise intensity (Stehly et al. 2006; Hillers et al. 2012). Friedrich et al. (1998) show that the noise wavefield is dominated by surface waves, with ratios between Love and Rayleigh waves changing with frequency. The wavefield associated with the microseisms is significantly less coherent than the one excited by earthquakes (e.g. Pedersen \& Krüger 2007), and its intensity much lower. By means of seismic interferometry, however, the approximate diffusivity of the noise wavefield can be exploited: given sufficiently long recordings of ambient seismic noise at two locations, group and phase velocity between these two locations can be extracted (Boschi \& Weemstra 2015, and citations therein).

Microseisms are most energetic in the period band between 5 and $30 \mathrm{~s}$. At the shorter period end of this range, surface waves from teleseismic earthquakes are difficult to observe. Local to regional seismicity is needed to obtain phase velocities from earthquake data in the frequency range between about 3 and $10 \mathrm{~s}$ (Endrun et al. 2004). Ambient noise can therefore provide information complementary to that obtained from earthquake-based studies (e.g. Meier et al. 2004; Lebedev et al. 2009; Yoshizawa \& Ekström 2010; Endrun et al. 2011; 
Agius \& Lebedev 2013). Because the frequency of a surface wave controls the depth range over which particle motion is excited, high-frequency signals contain information on the shallow part of the earth (e.g. Udías 1999), making ambient-noise studies ideal to assess the structure of the crust and upper mantle. Recordings of earthquake-generated teleseismic surface waves are dominated by lowerfrequency signal and thus sensitive to larger depths; their combination with ambient-noise data results in a data set that is sensitive to a broader depth range than ever previously achieved (Yao et al. 2006, 2008; Ritzwoller et al. 2011; Köhler et al. 2012; Zhou et al. 2012). We only treat surface-wave phase velocity in this work, which has the advantage that group velocity can be derived from it, while the opposite is not possible. Furthermore, phase velocities are to be preferred in tomographic inversions (e.g. Zhou et al. 2006).

Phase-velocity measurements made from ambient noise are only valid under certain conditions such as a uniform distribution of noise sources in space and time, a laterally homogeneous medium velocity, and far-field sources. As these conditions are not strictly met in practical situations, errors in the measured phase velocity are to be expected. These errors have been quantified for certain situations analytically and by synthetic models (Tsai 2009; Weaver et al. 2009; Yao \& Van Der Hilst 2009; Tsai \& Moschetti 2010; Tsai 2011). It is, however, not always possible to correct for them due to the complexity of these effects. It is therefore important to validate data obtained from ambient-noise analysis by comparison with equivalent measurements obtained from earthquake observations by classical methods. We focus here on ambient-noise reconstruction of Rayleigh- and Love-wave dispersion in Europe, comparing our observations with the two-station, earthquake-based dispersion database of Soomro et al. (2016).

A similar approach was followed before by Harmon et al. (2008), Yang et al. (2008a,b), Yao et al. (2008), Ritzwoller et al. (2011), Köhler et al. (2012), Zhou et al. (2012) and Shen et al. (2013). Yao et al. (2008) present a direct comparison of phase-velocity dispersion curves. The other authors compare mapped phase-velocity differences for specific regions. In all cases where differences have been analysed in detail, it is evident that velocities measured from ambient noise tend to be slightly lower, with reported differences between 0.07 and 0.001 $\mathrm{km} \mathrm{s}^{-1}$. This indicates that there might be a systematic effect which influences one or both of the methods.

The first objective of this work is to present the automated processing of ambient-noise data in the frequency domain. Our algorithm is based on the ideas of Aki (1957) and Ekström et al. (2009); it is essentially an improved version of the 'frequency domain' method employed by Boschi et al. (2013) and Korostelev et al. (2015), who did not describe in detail the underlying theory. In this approach, the zero crossings of the real part of the cross-correlation spectrum are evaluated to derive the phase-velocity dispersion. Importantly, this means that the Green's function's amplitude does not have to be reconstructed, which is the case when phase is to be determined by analysis of time-domain traces (Bensen et al. 2007; Lin et al. 2008; Zhou et al. 2012; Verbeke et al. 2012). We verify that our algorithm can successfully measure the phase velocity of both Love and Rayleigh waves from ambient noise. Ekström (2014), among others, already showed that Love-wave phase velocity can be determined from ambient noise. However, his data set was constructed in the assumption that wave polarization does not affect the data analysis procedure. We verify via synthetic tests that this approximation is acceptable at most periods and explain how to correct for it when taking measures at long periods.

The second objective is to validate ambient-noise observations by comparison with earthquake-based ones. We select central Europe as our study region to exploit the good station coverage found alone and around the Alpine chain. We compare the noise- and earthquake-based data at the same station couples in order to estimate the discrepancy in phase velocity from ambient-noise and earthquake records. The residuals are compared to known effects from noise theory and tested against synthetic experiments. By doing so, we prove the validity of results obtained from ambient noise and justify combining the two data sets in joint phase-velocity mapping.

\section{AMBIENT-NOISE THEORY}

\subsection{Vertical component (Rayleigh waves)}

Let us consider a Rayleigh wave in the frequency domain. The displacement is given, for example, by Aki \& Richards (2002), eq. (7.144). For now, we are only interested in the displacement along the $z$-component (perpendicular to the surface) and ignore all higher modes, assuming that the ambient-surface wavefield is dominated by the fundamental mode (Halliday \& Curtis 2008). Source and receiver are assumed to be at the surface so that the depth dependent eigenfunctions can be ignored. The amplitude is normalized according to Snieder (1986, 2004). With these simplifications the vertical displacement can be expressed as

$U_{1}(\omega)=A(\omega) e^{i \phi(\omega)} \sqrt{\frac{2 c(\omega)}{\pi \omega r_{1}}} e^{i\left(\frac{\omega r_{1}}{c(\omega)}+\frac{\pi}{4}\right)}$,

source 2-D Green's function

where $A(\omega)$ and $\phi(\omega)$ are the source's amplitude and phase spectra, $i$ is the imaginary unit, $\omega$ the angular frequency, $c(\omega)$ the dispersive phase velocity and $r_{1}$ is the distance travelled from the source to receiver 1. The expression for the Green's function is analogous to eq. (14) in Snieder (2004) and eq. (E17) in Boschi \& Weemstra (2015), except that we prefer here to employ the notation of Aki \& Richards (2002). The same wave recorded at a second station will have a slightly different phase and amplitude, according to the difference in distance travelled

$U_{2}(\omega)=A(\omega) e^{i \phi(\omega)} \sqrt{\frac{2 c(\omega)}{\pi \omega r_{2}}} e^{i\left(\frac{\omega r_{2}}{c(\omega)}+\frac{\pi}{4}\right)}$. 


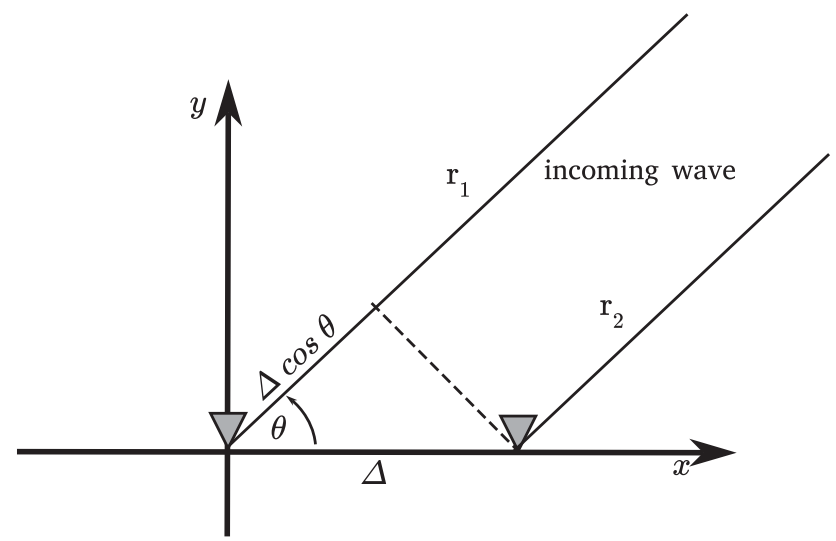

Figure 1. Incoming plane wave at an angle of $\theta$. The distance between the stations depicted as grey triangles is denoted by $\Delta$ and the difference in source-station path therefore coincides with $\Delta \cos \theta$.

The cross-correlation of the displacement at two stations located at $r_{1}$ and $r_{2}$, caused by a single source, is given, in the frequency domain, by multiplication with the complex conjugate (denoted by the superscript $*$ )

$U_{1}(\omega) U_{2}^{*}(\omega)=A^{2}(\omega) \frac{2 c(\omega)}{\pi \omega} \frac{1}{\sqrt{r_{1} r_{2}}} e^{i\left(\frac{\omega r_{1}}{c(\omega)}+\frac{\pi}{4}+\phi(\omega)\right)} e^{-i\left(\frac{\omega r_{2}}{c(\omega)}+\frac{\pi}{4}+\phi(\omega)\right)}=A^{2}(\omega) \frac{2 c(\omega)}{\pi \omega} \frac{1}{\sqrt{r_{1} r_{2}}} e^{i \frac{\omega}{c(\omega)}\left(r_{1}-r_{2}\right)}$.

For sources distributed along a circle of large radius $R$, measured from the centre of the coordinate system in Fig. 1, the expressions $r_{1} r_{2}$ and $r_{1}-r_{2}$ can be approximated according to Fig. 1,

$r_{1}-r_{2} \approx \Delta \cos \theta$

$r_{1} r_{2} \approx R(R-\Delta \cos \theta)=R^{2}\left(1-\frac{\Delta \cos \theta}{R}\right) \approx R^{2}$,

where $\Delta$ is the distance between the stations and $\theta$ is the angle towards the source. Strictly speaking, the above formula is only valid if sources are infinitely far away from the receivers, that is, for plane waves. The error introduced from this simplification will be discussed below with the help of synthetic tests. We now sum the cross-correlation for a large number of sources which we assume to correspond, approximately, to measuring the two-receiver cross-correlation over a long time period. Substituting the expressions from eq. (4) in eq. (3) and summing over $N$ sources gives

$C(\omega) \approx \frac{2 c(\omega)}{\pi \omega} \lim _{N \rightarrow \infty} \frac{1}{N}\left(\sum_{j}^{N} \frac{A_{j}^{2}(\omega)}{R} e^{i \frac{\omega}{c(\omega)} \Delta \cos \theta_{j}}\right)=\frac{2 c(\omega)}{\pi \omega R} \frac{1}{2 \pi} \int_{-\pi}^{\pi} A^{2}(\omega, \theta) e^{i \frac{\omega \Delta}{c(\omega)} \cos \theta} \mathrm{d} \theta$

$C(\omega)$ is the stacked cross-correlation resulting from an infinitely dense source distribution. Eq. (5) differs from its pure plane-wave counterpart in that the geometrical spreading term $1 / R$ is kept. We expressed the sum as an integral over all azimuths, where $A^{2}(\omega, \theta)$ is now an amplitude density depending on the azimuth $\theta$ of the incoming wave. Note that $A^{2}$ includes an implicit normalization dependent on the density of the discrete sources (i.e. it includes a constant with units $\mathrm{rad}^{-1}$ ), which ensures that the evaluation of the integral and the result of the summation give equal values and have equal units.

In case of simultaneously acting sources, constructive interference of signal coming from different sources gives rise to spurious traveltime delays. These spurious peaks in the cross-correlation are generally referred to as 'cross-terms' (e.g. Weemstra et al. 2015). Assuming the behaviour of different sources is uncorrelated, however, stacking over time renders these cross-terms negligible. Consequently, even if some of the $N$ sources in eq. (5) act simultaneously, the time-averaged cross-correlation will still coincide with the right-hand side of eq. (5). Summing cross-correlations over time is what is generally implemented in practice (e.g. Bensen et al. 2007).

\subsubsection{Exact integration}

We assume that the amplitude term in eq. (5) is independent of $\theta$, that is, there are sources with equal intensity everywhere around the receivers. It then follows that

$C(\omega) \approx \frac{2 c(\omega)}{\pi \omega R} \frac{1}{2 \pi} \int_{-\pi}^{\pi} A^{2}(\omega, \theta) e^{i \frac{\omega \Delta}{c} \cos \theta} \mathrm{d} \theta=\frac{2 c}{\pi \omega R} \frac{1}{2 \pi} A^{2}(\omega) \int_{-\pi}^{\pi} e^{i \frac{\omega \Delta}{c(\omega)} \cos \theta} \mathrm{d} \theta=\frac{2 c(\omega)}{\pi \omega R} A^{2}(\omega) J_{0}\left(\frac{\omega \Delta}{c(\omega)}\right)$,

where the definition of the Bessel function of the first kind and order $n$ from Abramowitz \& Stegun (1964) (eq. 9.1.21) has been used:

$J_{n}(z)=\frac{i^{-n}}{\pi} \int_{0}^{\pi} e^{i z \cos \theta} \cos (n \theta) \mathrm{d} \theta$. 
Eq. (6) is equivalent to eq. (72) in Boschi \& Weemstra (2015). The important result from eq. (6) is that cross-correlating the noise wavefield at two locations of known separation $\Delta$, gives an estimation of the phase velocity $c(\omega)$ between the stations (the detailed procedure is explained in the following sections). This relationship was first derived by Aki (1957) and is at the basis of the SPAC (spatial autocorrelation) method (Aki 1957; Bettig et al. 2001) for local arrays. The equivalence of temporal (as in this work) and spatial cross-correlation (as in the SPAC method) is discussed by Tsai \& Moschetti (2010).

We obtained eq. (6) in the assumption that $A$ is independent of the direction of propagation. For a strongly non-uniform illumination pattern, the deviations from a Bessel function can be quite large and the imaginary part will not vanish (Tsai 2009; Weaver et al. 2009; Yao \& Van Der Hilst 2009; Basini et al. 2013). This can result in erroneous phase velocities. In most applications, however, this approximation is good enough. Examples using synthetic tests are shown below.

\subsubsection{Stationary-phase integration}

It is also possible to solve the integral in eq. (6) by a stationary-phase approximation (e.g. Snieder 2004; Boschi \& Weemstra 2015). In this case, $\omega$ and/or $\Delta$ is assumed to be large, which means that $\exp (i(\omega \Delta \cos \theta / c))$ is a rapidly oscillating function of $\theta$. A rapid oscillation around zero means equal positive and negative contributions to the integral over $\theta$ which will approximately cancel out. It is thus enough to evaluate the integral at the stationary points where the phase term $\psi(\theta)=\Delta \cos \theta / c$ is stationary, that is, $\mathrm{d} \psi(\theta) / \mathrm{d} \theta=0$. This gives

$I(\omega)=\int_{-\infty}^{\infty} f(\theta) e^{i \omega \psi(\theta)} \mathrm{d} \theta \approx f(a) e^{i\left(\omega \psi(a) \pm \frac{\pi}{4}\right)} \sqrt{\frac{2 \pi}{\omega\left|\psi^{\prime \prime}(a)\right|}}$,

for a single stationary point at $a$, valid for $\omega \rightarrow \infty$ and assuming that the second derivative $\psi^{\prime \prime}$ with respect to $\theta$ is not zero at $\theta=a$ (e.g. Bender \& Orszag 1978). The sign of $\pi / 4$ in the exponent is positive for $\psi^{\prime \prime}(a) \geq 0$ and negative otherwise. If we have a definite integral and the stationary point is at one of the integration limits, a factor of $\frac{1}{2}$ has to be included. In eq. (6), there are three stationary points at

$\frac{\mathrm{d}}{\mathrm{d} \theta} \frac{\Delta \cos \theta}{c(\omega)}=-\frac{\Delta \sin \theta}{c(\omega)}=0$, for $\theta=-\pi, 0, \pi$,

with two of the stationary points at the integration limits. We can thus solve the integral in eq. (6),

$$
\begin{aligned}
C(\omega) \approx & \frac{2 c(\omega)}{\pi \omega R} \frac{1}{2 \pi} \int_{-\pi}^{\pi} A^{2}(\omega, \theta) e^{i \frac{\omega \Delta}{c(\omega)} \cos \theta} \mathrm{d} \theta \approx \frac{c(\omega)}{\pi^{2} \omega R}\left[\frac{1}{2} A^{2}(\omega,-\pi) \sqrt{\frac{2 \pi}{\frac{\omega \Delta}{c(\omega)}}} e^{-i\left(\frac{\omega \Delta}{c(\omega)}-\frac{\pi}{4}\right)}\right. \\
& \left.+A^{2}(\omega, 0) \sqrt{\frac{2 \pi}{\frac{\omega \Delta}{c(\omega)}}} e^{i\left(\frac{\omega \Delta}{c(\omega)}-\frac{\pi}{4}\right)}+\frac{1}{2} A^{2}(\omega, \pi) \sqrt{\frac{2 \pi}{\frac{\omega \Delta}{c(\omega)}}} e^{-i\left(\frac{\omega \Delta}{c(\omega)}-\frac{\pi}{4}\right)}\right] .
\end{aligned}
$$

Since $A(\omega,-\pi)=A(\omega, \pi)$, we can write

$C(\omega) \approx \frac{c(\omega)}{\pi^{2} \omega R}\left[A^{2}(\omega, 0) \sqrt{\frac{2 \pi}{\frac{\omega \Delta}{c(\omega)}}} e^{i\left(\frac{\omega \Delta}{c(\omega)}-\frac{\pi}{4}\right)}+A^{2}(\omega, \pi) \sqrt{\frac{2 \pi}{\frac{\omega \Delta}{c(\omega)}}} e^{-i\left(\frac{\omega \Delta}{c(\omega)}-\frac{\pi}{4}\right)}\right]$.

The terms $\exp \left( \pm i\left(\frac{\omega \Delta}{c(\omega)}-\frac{\pi}{4}\right)\right)$ describe a wave travelling from receiver 1 to receiver 2 and vice versa, which are often referred to as causal and anti-causal parts of the cross-correlation.

Eq. (11) shows that the contributions to the cross-correlation associated with sources at $\theta=0$ and $\theta=\pi$ have phases of $\omega \Delta / c-\pi / 4$ and $\omega \Delta / c+\pi / 4$, respectively. This $\pi / 4$ phase shift was something being thought of as a 'discrepancy' between causal/acausal terms and the phase of an impulse travelling with speed $c$ between the locations. Due to the fact that we are dealing with a circular source distribution, the solution is characterized by a Bessel function (eq. 6). In the high-frequency and/or long interstation distance limit, the Bessel function is approximated by a cosine with $\pi / 4$ phase shift (Fig. 2). This explains the stationary-phase solution in eq. (11). Most time-domain approaches use this approximation and consequently it is important to apply the $\pi / 4$ correction. However, this introduces an error at low frequencies and/or short interstation distance (Tsai 2009; Boschi et al. 2013). The frequency-domain algorithm presented here does not require such correction as we determine phase velocities using directly the properties of the Bessel function.

The source intensity $A$ in eq. (11) is allowed to be a smoothly varying function of $\theta$. We can see that the forward and backward terms are scaled by the amplitude term in 0 - and $\pi$-direction, that is, aligned with the station pair (Fig. 1). This means that in the infinite frequency limit, only sources on the $x$-axis contribute constructively. For lower frequencies, the Fresnel zone is characterized by two hyperbolae aligned with the $x$-axis (e.g. Snieder 2004). The azimuths where noise sources contribute constructively can be expressed in an approximate formula from Yao \& Van Der Hilst (2009)

$|\Delta \cos (\theta)-\Delta|<\frac{c(\omega)}{2 \omega}$,

referring to the definition of $\Delta$ and $\theta$ from Fig. 1.

Note that in the stationary-phase approach, eq. (11) can be derived as well without the plane wave approximations from eq. (4) (Boschi \& Weemstra 2015). 


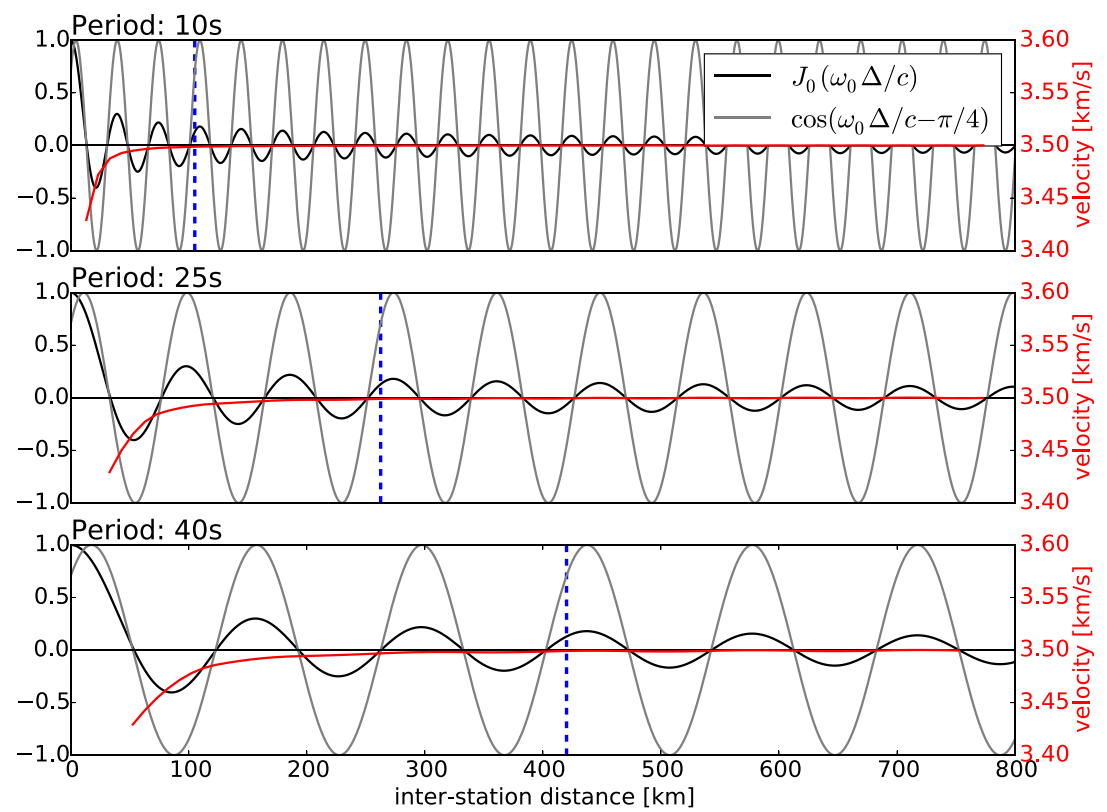

Figure 2. Bessel function (black line) compared to its high-frequency/long interstation distance approximation (grey), which is often used in time domain approaches. Both curves are shown with respect to interstation distance and at three different periods. The velocity $c$ is set to $3.5 \mathrm{~km} \mathrm{~s}{ }^{-1}$ for both curves. In time domain approaches, the phase velocity is determined by evaluating the phase of the stacked cross-correlation spectrum and correcting it by $\pi / 4$. This introduces an error at long periods and short interstation distances, plotted here as red curve. Accordingly, data associated with short (typically less than three wavelengths, blue dashed line) interstation distance are often discarded (e.g. Bensen et al. 2007).

Using $i=\exp (i \pi / 2)$, eq. (11) can be rewritten as

$C(\omega) \approx \frac{c(\omega)}{i \pi \omega R}\left(A^{2}(\omega, 0) \sqrt{\frac{2 c(\omega)}{\pi \omega \Delta}} e^{i\left(\frac{\omega \Delta}{c(\omega)}+\frac{\pi}{4}\right)}-A^{2}(\omega, \pi) \sqrt{\frac{2 c(\omega)}{\pi \omega \Delta}} e^{-i\left(\frac{\omega \Delta}{c(\omega)}+\frac{\pi}{4}\right)}\right)$,

which contains the far-field approximation of the Hankel function (eq. C4-5 in Boschi \& Weemstra 2015), yielding

$C(\omega) \approx \frac{c(\omega)}{\pi \omega R}\left[A^{2}(\omega, 0)\left(J_{0}\left(\frac{\omega \Delta}{c(\omega)}\right)+i Y_{0}\left(\frac{\omega \Delta}{c(\omega)}\right)\right)+A^{2}(\omega, \pi)\left(J_{0}\left(\frac{\omega \Delta}{c(\omega)}\right)-i Y_{0}\left(\frac{\omega \Delta}{c(\omega)}\right)\right)\right]$.

If we assume as before, that $A(\omega, \theta)$ is equal for all directions we get

$C(\omega) \approx \frac{2 c(\omega)}{\pi \omega R} A^{2}(\omega) J_{0}\left(\frac{\omega \Delta}{c(\omega)}\right)$

which is the same as eq. (6). It is clear from the above treatment that if $A(0) \neq A(\pi)$ the imaginary part of the cross-correlation spectrum does not vanish. From eq. (14), it may also look like that in this case the real part of the cross-correlation spectrum still follows a Bessel function. However, this is strictly speaking only true in the high frequency limit, for which the stationary-phase result was derived.

\subsection{Horizontal components (Rayleigh and Love waves)}

When applying this method to Love waves, we are interested in the horizontal displacement, perpendicular to the great-circle identified by the receiver locations ( $y$-axis in Fig. 1). With the same assumptions as for eq. (1), eq. (7.143) from Aki \& Richards (2002) can be simplified as

$U_{1 L}(\omega)=A_{L}(\omega) \sqrt{\frac{2 c_{L}(\omega)}{\pi \omega r_{1}}} e^{i\left(\frac{\omega r_{1}}{c_{L}(\omega)}+\frac{\pi}{4}+\phi(\omega)\right)} \cos (\theta)$,

which is the same as eq. (1), apart from the $\cos (\theta)$ term, where $\theta$ is the angle from station 1 towards the source (Fig. 1). Hence, the $\cos (\theta)$ term assures that, for Love waves and the geometry in Fig. 1, we are only looking at the $y$-component of the displacement. The radial (parallel to the receiver-receiver great circle) Rayleigh-wave displacement has the same expression, with $A_{R}$ and $c_{R}$ replacing $A_{L}$ and $c_{L}$, respectively. With the same far-field assumptions as above (meaning also that $\theta$ is equal for both stations), the cross-correlation of horizontal surface-wave displacement at the two stations reads

$C_{h}(\omega) \approx \frac{2 c(\omega)}{\pi \omega R} A^{2}(\omega) \frac{1}{2 \pi} \int_{-\pi}^{\pi} e^{i \frac{\omega \Delta}{c(\omega)} \cos \theta} \cos ^{2}(\theta) \mathrm{d} \theta$, 

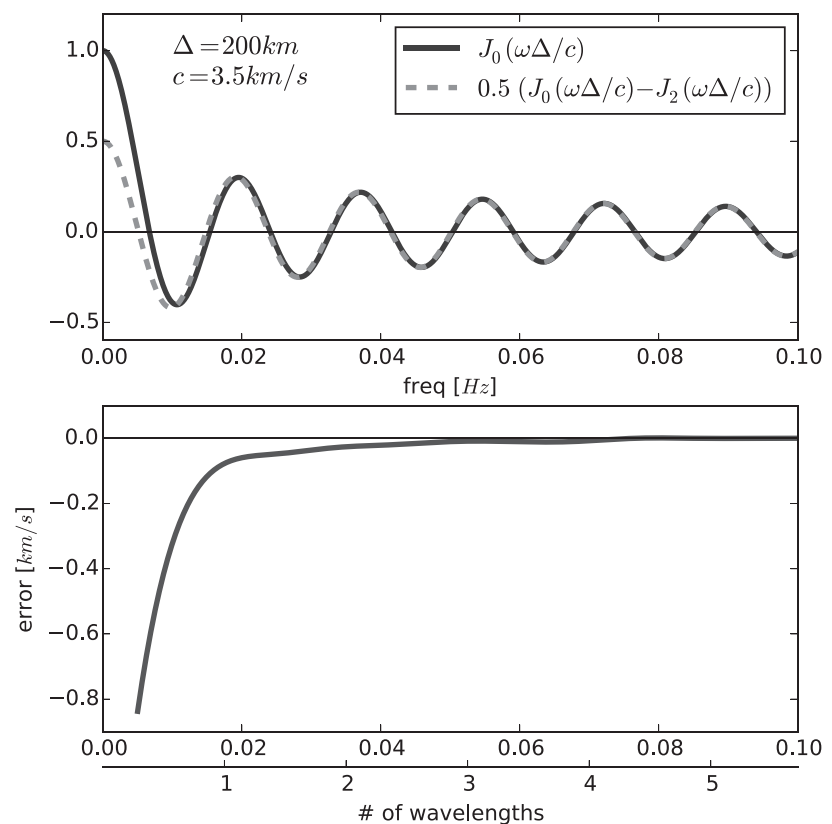

Figure 3. Top panel: zero-order Bessel function $J_{0}$ of $\frac{\omega \Delta}{c}$, and difference between $J_{0}$ and the second-order Bessel function $J_{2}$ of the same argument. These functions control the vertical- and horizontal-component cross-correlations according to eqs (15) and (19), respectively, for the particular case of constant $c=3.5 \mathrm{~km} \mathrm{~s}^{-1}$ (no dispersion). In the literature, the contribution of $J_{2}$ to the horizontal component is sometimes neglected, resulting in an error that can be significant at low frequencies and/or short interstation distances. We quantify this error by applying our measurement algorithm on the cross-correlations of the top panel, and plotting (bottom panel) the difference between the resulting dispersion curves. The secondary $x$-axis of the bottom plot shows the number of wavelengths that correspond to an interstation distance of $200 \mathrm{~km}$ and a phase velocity of $3.5 \mathrm{~km} \mathrm{~s}^{-1}$ at the respective frequency.

where $A$ and $c$ have to be adapted for Rayleigh and Love waves, accordingly. Eq. (17) can be seen as the radial- and transverse counterpart of eq. (6). Making use of the trigonometric identity for $\cos ^{2}(\theta)=(\cos (2 \theta)+1) / 2$ and rearranging yields

$$
\begin{aligned}
C_{h}(\omega) & \approx \frac{2 c(\omega)}{\pi \omega R} A^{2}(\omega) \frac{1}{2 \pi} \int_{-\pi}^{\pi} e^{i \frac{\omega \Delta}{c(\omega)} \cos \theta} \frac{1+\cos (2 \theta)}{2} \mathrm{~d} \theta \\
& =\frac{c(\omega)}{\pi \omega R} A^{2}(\omega) \frac{1}{2 \pi}\left[\int_{-\pi}^{\pi} e^{i \frac{\omega \Delta}{c(\omega)} \cos \theta} \mathrm{d} \theta+\int_{-\pi}^{\pi} e^{i \frac{\omega \Delta}{c(\omega)} \cos \theta} \cos (2 \theta) \mathrm{d} \theta\right] .
\end{aligned}
$$

After substituting eq. (7) into eq. (18), the latter equation takes the simpler form

$C_{h}(\omega) \approx \frac{c(\omega)}{\pi \omega R} A^{2}(\omega)\left[J_{0}\left(\frac{\omega \Delta}{c(\omega)}\right)-J_{2}\left(\frac{\omega \Delta}{c(\omega)}\right)\right]$.

This result is also given by Aki (1957) and Sánchez-Sesma \& Campillo (2006).

Alternatively, the integral in eq. (17) can be solved via the stationary-phase approximation, since $\cos ^{2}(\theta)$ is smooth relative to the rapid oscillation of the phase term for high $\omega$. This gives for the three stationary points at $-\pi, 0$ and $\pi$

$$
\begin{aligned}
C_{h}(\omega) \approx & \frac{2 c(\omega)}{\pi \omega R} \frac{1}{2 \pi} \int_{-\pi}^{\pi} A^{2}(\omega, \theta) e^{i \frac{\omega \Delta}{c(\omega)} \cos \theta} \cos ^{2}(\theta) \mathrm{d} \theta \approx \frac{c(\omega)}{\pi^{2} \omega R}\left[\frac{1}{2} A^{2}(\omega,-\pi) \cos ^{2}(-\pi) \sqrt{\frac{2 \pi}{\frac{\omega \Delta}{c(\omega)}}} e^{-i\left(\frac{\omega \Delta}{c(\omega)}-\frac{\pi}{4}\right)}\right. \\
& \left.+A^{2}(\omega, 0) \cos ^{2}(0) \sqrt{\frac{2 \pi}{\frac{\omega \Delta}{c(\omega)}}} e^{i\left(\frac{\omega \Delta}{c(\omega)}-\frac{\pi}{4}\right)}+\frac{1}{2} A^{2}(\omega, \pi) \cos ^{2}(\pi) \sqrt{\frac{2 \pi}{\frac{\omega \Delta}{c(\omega)}}} e^{-i\left(\frac{\omega \Delta}{c(\omega)}-\frac{\pi}{4}\right)}\right] \\
= & \frac{2 c(\omega)}{\pi \omega R} A^{2}(\omega) J_{0}\left(\frac{\omega \Delta}{c(\omega)}\right),
\end{aligned}
$$

if we assume, as above, that $A(0)=A(\pi)$. The right-hand side of eq. (20) coincides with that of eq. (15) (e.g. Snieder 2004) and differs from the exact integration in eq. (19). This is consistent with the high frequency behaviour of eq. (19), which we have verified numerically as shown in Fig. 3, that is,

$$
\lim _{\omega \rightarrow \infty} \frac{1}{2}\left[J_{0}\left(\frac{\omega \Delta}{c(\omega)}\right)-J_{2}\left(\frac{\omega \Delta}{c(\omega)}\right)\right]=J_{0}\left(\frac{\omega \Delta}{c(\omega)}\right) .
$$

Estimating phase velocities of Love waves or the horizontal component of Rayleigh waves with a simple Bessel function will therefore introduce an error, but only at low frequencies. An example of this effect is shown in Fig. 3. Most researchers do not apply the ambient-noise method (ANM) at periods longer than $30 \mathrm{~s}(0.033 \mathrm{~Hz})$, where the discrepancy shown in Fig. 3 is lower than $0.03 \mathrm{~km} \mathrm{~s}^{-1}(0.9$ per cent) for an 
interstation distance of $200 \mathrm{~km}$. With increasing interstation distance $\Delta$ this effect reduces further. Discarding all data where the interstation distance is shorter than three wavelengths (e.g. Bensen et al. 2007) reduces the error to 0.25 per cent over all frequencies. Consequently, this issue is often ignored when extracting Love waves (e.g. Lin et al. 2008; Ekström 2014). However, Fig. 3 shows that at low frequencies the error can be as large as 10-20 per cent, which corresponds to typically mapped peak-to-peak phase-velocity differences in the earth (e.g. Verbeke et al. 2012; Ekström 2014; Soomro et al. 2016). As we aim to exploit all available data and extend the limits of the method to lower frequencies, we include this modification in the presented procedure.

\subsection{Pre-processing and correlation}

Our algorithm measures the phase-velocity curve for a given station couple by cross-correlation and stacking of noise records. It is applied to pairs of sufficiently long seismograms, which ensures that signals from a relatively large number of sources at different azimuths are recorded. At regional/continental scale, it is preferable to work with one-year-long recordings, since the azimuth of noise propagation has been shown to change seasonally (e.g. Stehly et al. 2006; Yang \& Ritzwoller 2008). For the same reason, the benefit of using more than one year of data with respect to one full year might be negligible. More detailed discussions on the time span of noise recordings can be found in Bensen et al. (2007) and Seats et al. (2012).

Records are filtered in the frequency band of interest (2-200s). First, this allows decimation (reduction of sample rate) for all seismograms where $1 \mathrm{~Hz}$ sampling rate data are not readily available, which saves disk space and speeds up processing. Second, it restricts the following restitution filtering to a valid frequency range. In our case, pre-processing includes removing the instrument response (restitution), to avoid that different phase responses of used seismometers change the phase-velocity measurement. However, broad-band instruments have often a zero phase shift over a large frequency range and our tests show accordingly that the effects from restitution are marginal.

Wherever gaps appear in the recordings we zero-pad the data in order to get continuous time series. The data is then cut into overlapping time windows (e.g. half an hour with 50 per cent overlap), cosine-tapered at both ends and Fourier transformed. The effects of different window lengths and overlaps are discussed by Seats et al. (2012). Spectral whitening (e.g. Bensen et al. 2007) equalizes all amplitudes, therefore broadening the spectrum, downweighting strong signals from earthquakes and reducing influence from monochromatic sources:

$C_{\text {whitened }}(\omega)=\frac{U_{1}(\omega) U_{2}^{*}(\omega)}{\left|U_{1}(\omega)\right|\left|U_{2}(\omega)\right|}$

There are also other approaches to achieve this (e.g. Bensen et al. 2007; Ekström et al. 2009); Ekström (2014), however, notes that effects of whitening or removal of earthquake signals have only very small influence on the phase-velocity measurement.

This procedure is applied to the Z- or R-component recordings for Rayleigh waves and T-component recordings for Love waves. The $\mathrm{R}$ direction is defined as parallel to the great circle connecting the station pair; $\mathrm{T}$ is perpendicular to both $\mathrm{R}$ and $\mathrm{Z}$.

\subsection{Phase-velocity determination}

In the following, we discard the imaginary part of the stacked cross-correlation spectrum which should ideally be zero (eq. 14). Its real part is often characterized by strong high-frequency variability that causes additional, spurious zero crossings (Fig. 4). This can be overcome by a smoothing filter, in our case a simple low-pass filter. The corner frequency depends on the interstation distance and on estimated minimum phase velocity. In the next step, the zero crossings of the smoothed curve are identified and compared to the Bessel function.

Let us denote $z_{m}(m=1,2, \ldots, \infty)$ the discrete set of values of the argument of $J_{0}$, such that $J_{0}\left(z_{m}\right)=0$. Eq. (6) shows that the vertical-component ambient-noise cross-correlation coincides with the Bessel function $J_{0}(\omega \Delta / c(\omega))$. By estimating a realistic minimum value for the phase velocities $c(\omega)$, we can reduce $z_{m}$ to a finite set, $m=1,2, \ldots, M$. Ekström et al. (2009) proposed to estimate the dispersion curve $c(\omega)$ from the observed ambient-noise cross-correlation, by simply identifying the values $\omega_{n}$ of $\omega$ for which the observed cross-correlation is zero. Each $\omega_{n}$ corresponds to a discrete frequency where the spectrum is zero.

$\frac{\omega_{n} \Delta}{c\left(\omega_{n}\right)}=z_{m}$

from which we can derive all possible phase velocities as

$c\left(\omega_{n}\right)=\frac{\omega_{n} \Delta}{z_{m}}$,

giving $M$ possible phase velocities corresponding to $\omega_{n}$. This number of possible values can be reduced by one half by only taking into account the zero crossings where the derivative of the Bessel function has the same sign as that of the (real part of the) observed spectrum. The problem is further simplified by limiting the result to a range of realistic phase velocities for the region, which is especially helpful at long periods where only a few points to choose from remain. To measure Love-wave phase velocity, or to have an alternative estimate of Rayleigh wave phase velocity via the radial component, the zero crossings of $J_{0}(z)-J_{2}(z)$ have to be determined; recall, however, that at high frequencies those coincide approximately with the zero crossings of $J_{0}(z)$.

In Fig. 4(b), an example of the real part of a cross-correlation spectrum and its smoothed version are shown. For each of the zero crossings at the frequencies $\omega_{n}$, all of the $M$ resulting phase velocities are plotted in Fig. 4(c). The goal is now to implement an automated 
(a)

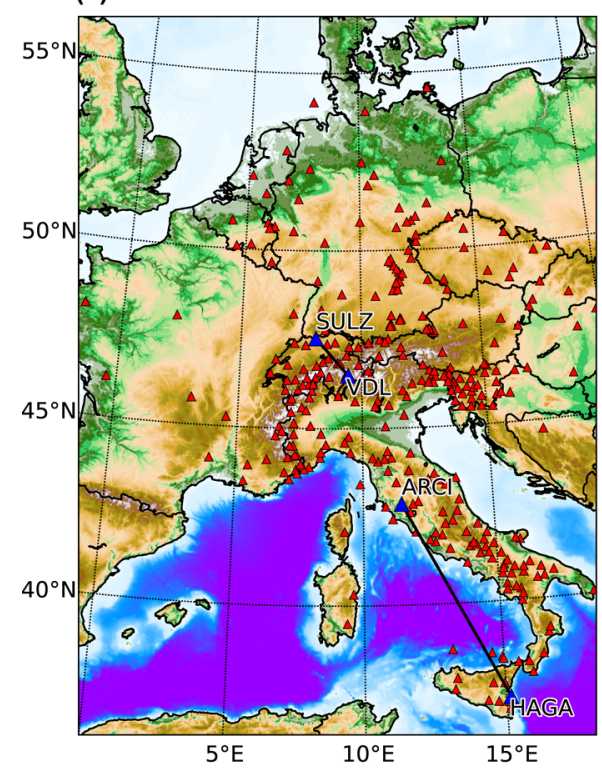

(b)

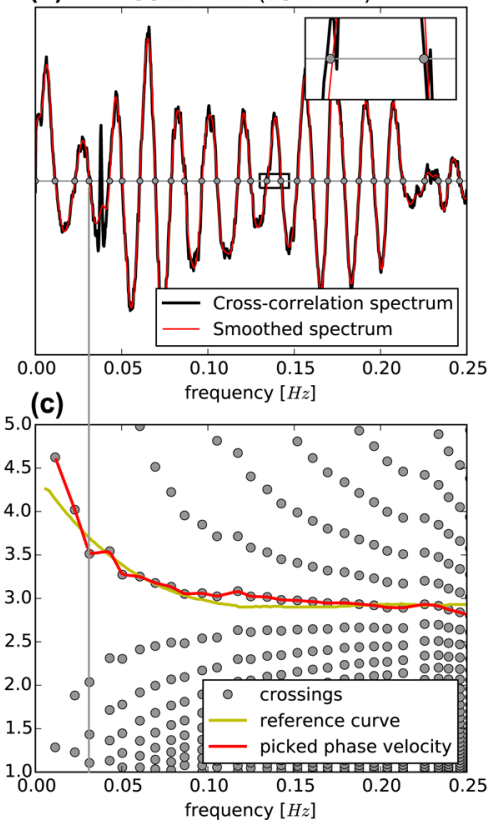

(d) $\quad$ ARCI - HAGA $(692.4 \mathrm{~km})$
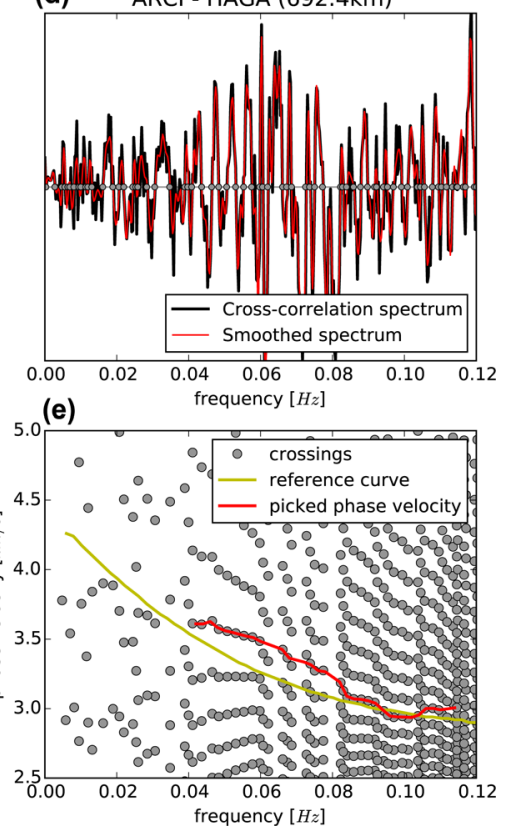

Figure 4. Processing of ambient-noise cross-correlation spectrum. (a) Station map. The selected stations for the examples are highlighted in blue. (b) Real part of the stacked cross-correlation spectrum for an approx. $1000 \mathrm{~d}$ record at SULZ and VDL before (black) and after (red) smoothing. A clear peak at $0.038 \mathrm{~Hz}$ is visible which does not fit the period of the rest of the spectrum. It is ignored by the smoothing as it would cause a velocity jump in the phase-velocity measurement. The inset zooms in on two zero crossings, where smoothing prevents additional zero crossings that are caused by unwanted noise in the spectrum. (c) Phase velocities from the spectrum's zero crossings. Each spectrum's zero crossing can be explained by several possible phase velocities as indicated by the grey line crossing between plots (b) and (c). The reference model is shown as yellow curve. The phase velocities are picked successively starting from the lowest frequencies. (d) Same as (b) for station couple ARCI and HAGA. The higher frequency of oscillation in the spectrum is due to the larger interstation distance. Only $200 \mathrm{~d}$ of correlation are available which causes a very noisy spectrum. (e) The low data quality makes phase-velocity picking difficult. Automated picks are only taken in a very limited frequency range [note the different scales of the frequency axis between (c) and (e)].

procedure that chooses the most realistic dispersion curve $c(\omega)$ by picking the right phase velocities. The least ambiguity is found at the low frequency end, where only few possible phase velocities fall within a realistic range. In order to choose the most likely value, the algorithm relies on a reference model (yellow curve in Fig. 4c). This model may come from a reference earth model, previous studies or by manual picking the dispersion curve for a couple of station pairs that represent a good average in the region of interest.

At the low frequency end, the automated picking relies only on this reference curve and will choose the phase velocities that are closest to it. After the first few values have been determined, more criteria are taken into account. Such criteria are defined based on the properties of Bessel functions:

(i) The frequency step $d \omega$ from one zero crossing to the next is approximately $\pi$, which becomes clear when one looks at the asymptotic approximation of the Bessel function by a cosine with $\pi / 4$ phase shift. So when choosing the next phase-velocity value at frequency $\omega_{k+1}$, it should be verified that

$d \omega=\omega_{k+1}-\omega_{k} \approx \pi \frac{c\left(\omega_{k}\right)}{\Delta}$

assuming that $c\left(\omega_{k}\right)$ is not too different from $c\left(\omega_{k+1}\right)$. This should be the case since surface-wave velocity implicitly averages, and thus smooths, Earth structure over depth. If the frequency step $d \omega$ is much smaller than the prediction, the spectrum is probably noisy, resulting in spurious zero crossings and therefore a jump to a higher phase velocity. Consequently, values of $\omega_{n}$ are ignored (up to an acceptable threshold) until a zero crossing is found whose frequency has an acceptable frequency-step width. On the other hand it can also happen that the spectrum does not have any zero crossing in a realistic range. This causes the algorithm to stop, because it is not possible to choose the next phase-velocity value without ambiguity.

(ii) In Fig. 4(c), several branches are visible that are sub-parallel to the red curve. The existence of these branches is linked to the ambiguity in $m$. The velocity jump from one branch to the next, that is, $z_{m}$ to $z_{m \pm 2}$ in eq. (24), is associated with $2 \pi\left(z_{m \pm 1}\right.$ has another crossing direction as $z_{m}$ and can therefore be excluded). As phase velocities are supposed to vary smoothly, only picks that are at a certain threshold below $2 \pi$ are considered.

Each picked phase-velocity value has to comply with the two above criteria. After a couple of points have been picked, a linear extrapolation over the last picks predicts the next possible value for the dispersion curve. Additional stabilizing criteria are a comparison of the gradient of the picked curve with the reference model, for example when steeply increasing phase velocities are picked while the model indicates rather decreasing values. The algorithm also tries to choose automatically where to start the picking of the phase-velocity curve, 
(a)

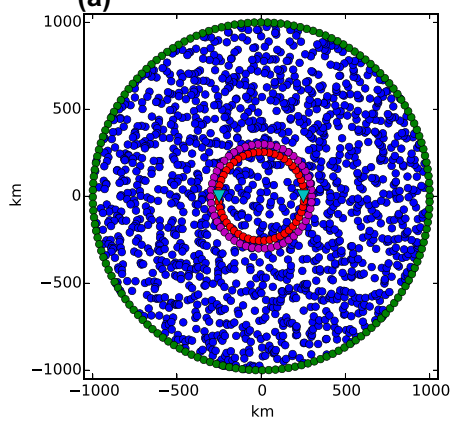

(b)

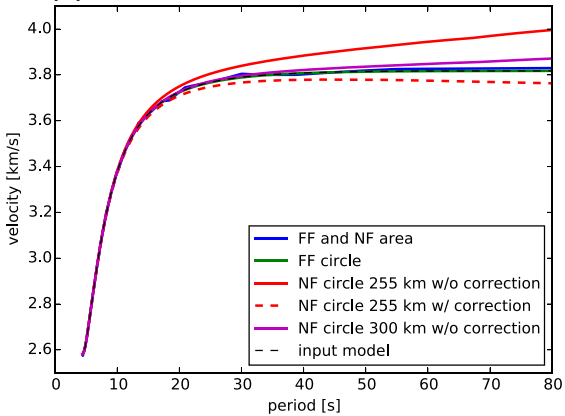

(c)

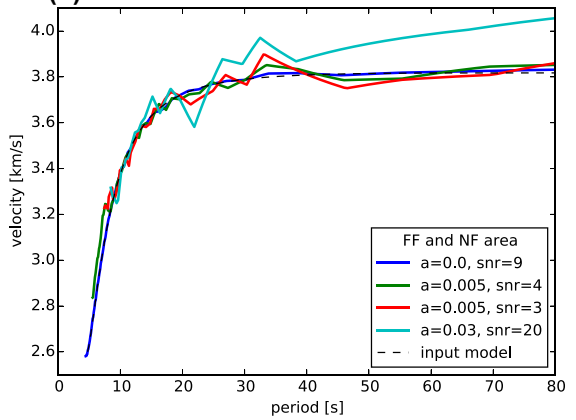

Figure 5. Phase-velocity estimation for four different scenarios of synthetic source distributions. (a) Map view of two stations at $500 \mathrm{~km}$ distance (triangles) and four example distributions of noise sources are shown. Blue circles: circular area of randomly distributed noise sources; green circles: sources in the far-field (FF); red circles: sources in the near field (NF), $255 \mathrm{~km}$ radius; magenta circles: sources in the near field, $300 \mathrm{~km}$ radius. (b) Respective phase-velocity estimations are shown in comparison with the dispersive input-velocity model. For explanation of the correction applied to the near-field sources, see the text. (c) Synthetic phase-velocities for the random source distribution in (a). $a$ is the attenuation factor and snr is the signal-to-noise ratio.

when the cross-correlation spectrum is very noisy at low frequencies. For example, in Fig. 4(e), it only starts at $0.04 \mathrm{~Hz}$ and stops at $0.11 \mathrm{~Hz}$, because the $d \omega$ criterion (i) has been violated too often.

\section{SYNTHETIC TESTS OF SOURCE DISTRIBUTION EFFECTS}

In this section, a station pair is placed in a simple medium with homogeneous velocity. Point sources are distributed in a number of ways around the station couple. Each source has a random amplitude and phase spectrum and the signal at the station is calculated by convolution with the 2-D Green's function (e.g. Boschi \& Weemstra 2015, E15). The synthetic signals are cross-correlated for each source and the cross-correlation spectrum is stacked. No additional random error is applied to the data. The synthetic data are cross-correlated and evaluated in the same way as the real data, as explained in the previous section, in order to obtain a phase-velocity curve.

\subsection{Far-/near-field sources}

In Fig. 5, we compare the effects of far- and near-field source distributions. We use a dispersive phase-velocity distribution as 'input model'. We first assume a far-field source distribution covering all azimuths (green circles in Fig. 5a), reproducing the idealized setup assumed in the analytical derivation of Section 2. A source-receiver distance of $1000 \mathrm{~km}$ corresponds to 3.3 wavelengths at a period of $80 \mathrm{~s}$ and velocity of $3.8 \mathrm{~km} \mathrm{~s}^{-1}$, which is large enough to meet the far-field criteria and indeed, the dispersion curve is perfectly recovered as shown in Fig. 5(b). In this setup, the maximum azimuth difference for a given source amounts to $28^{\circ}$.

In a second test, we assume again a circular source distribution, but with the circle lying in the near field of the receivers (red circles in Fig. 5a). The resulting discrepancy between input and output dispersion curves, illustrated in Fig. 5(b), can be explained in terms of the approximations made in the derivation of Section 2.1, which can be summarized as follows:

(i) to simplify our forward calculations, we have made use of a far-field approximation of the Green's function (eq. 1);

(ii) eq. (4) is valid for far-field sources (plane waves) only;

(iii) the stationary phase approximation in eq. (11) is valid only for high frequencies and/or large interstation distance $\Delta$.

Point (iii) is not relevant to the rest of this study because our algorithm does not rely on the stationary-phase solution but uses the solution of the exact integration, that is, a Bessel function (eq. 6 or 15). The problem arising from (ii) is associated with the fact that in the near field the distance difference from source to station 1 and source to station 2 is generally smaller than what the far-field approximation requires (eq. 4). It is possible to correct for this effect by taking the exact definition of $r_{1}-r_{2}$ which invokes, however, an estimation of the average distance $R$ of sources providing the main contribution to observations. It is thus necessary to repeat the derivation that lead to eq. (5) without the far-field approximations from eq. (4). Again, we assume a circular source distribution, but with sources at a relatively small radius $R$. We find,

$C(\omega)=\frac{2 c(\omega)}{\pi \omega} \frac{1}{2 \pi} \int_{-\pi}^{\pi} \frac{A^{2}(\omega, \theta)}{\sqrt{r_{1}(\theta) r_{2}(\theta)}} e^{i \frac{\omega}{c(\omega)}\left(r_{1}(\theta)-r_{2}(\theta)\right)} \mathrm{d} \theta$.

According to Fig. 1, we can write

$$
\begin{gathered}
r_{1}(\theta)-r_{2}(\theta)=R-\left[(R \cos \theta-\Delta)^{2}+(R \sin \theta)^{2}\right]^{1 / 2}=R-\left[\Delta^{2}-2 \Delta R \cos \theta+R^{2}\right]^{1 / 2}, \\
r_{1}(\theta) r_{2}(\theta)=R\left[(R \cos \theta-\Delta)^{2}+(R \sin \theta)^{2}\right]^{1 / 2}=R\left[\Delta^{2}-2 \Delta R \cos \theta+R^{2}\right]^{1 / 2},
\end{gathered}
$$


which we substitute into eq. (26):

$C(\omega)=\frac{2 c(\omega)}{\pi \omega} \frac{1}{2 \pi} \int_{-\pi}^{\pi} \frac{A^{2}(\omega, \theta)}{\sqrt{R\left[\Delta^{2}-2 \Delta R \cos \theta+R^{2}\right]^{1 / 2}}} e^{i \frac{\omega}{c(\omega)}\left(R-\left[\Delta^{2}-2 \Delta R \cos \theta+R^{2}\right]^{1 / 2}\right)} \mathrm{d} \theta$.

We now define another function $f(x)$ which is independent of $c(\omega)$,

$f(x)=\frac{2}{\pi \omega} \frac{1}{2 \pi} \int_{-\pi}^{\pi} \frac{A^{2}(\omega, \theta)}{\sqrt{R\left[\Delta^{2}-2 \Delta R \cos \theta+R^{2}\right]^{1 / 2}}} e^{i \omega\left(R-\left[\Delta^{2}-2 \Delta R \cos \theta+R^{2}\right]^{1 / 2}\right)} \mathrm{d} \theta$.

For a given station couple with interstation distance $\Delta$, making a reasonable guess for $R$ and assuming an idealized source distribution $A(\omega, \theta)$, which is 1 for all $\theta$, we can integrate the right hand side of eq. (29) numerically. We can then extract (also numerically) the zero crossings $z_{m}$ of $f(x)$. The following procedure is the same as described in Section 2.4. Each zero-crossing of the data-derived spectrum $C(\omega)$ is compared to the zero crossings of the near-field corrected function $f(x)$. All possible phase velocities $c_{m}\left(\omega_{0}\right)$ for a zero crossings in $C(\omega)$ at $\omega_{0}$ are given by

$c\left(\omega_{0}\right)=\frac{\omega_{0}}{z_{m}}$.

This is the same as eq. (24), but without $\Delta$, which we already took into account when we integrated eq. (29) numerically.

If the signal is whitened prior to cross-correlation (eq. 22) and if sources are not acting simultaneously, the $\frac{A^{2}(\omega, \theta)}{\sqrt{r_{1}(\theta) r_{2}(\theta)}}$ term in eq. (26) cancels out. This has to be taken into account when integrating eq. (29). This approach results in the dashed red line of Fig. 5.

An analytical near-field correction is proposed by Tsai (2009) which is, however, only valid if $\Delta / R \leq 1$, which is not the case in the presented example. His approximation also requires an estimation of $R$.

The remaining difference between the dashed red line in Fig. 5 and the input model is due to (i): the far-field approximation of the Green's function. Note also that in the test setup we worked with point sources which is unlikely to be realistic for longer period waves. It is clear from Fig. 5 that the near-field effects are only important at long periods. Additional tests (not shown here) indicate that the near-field error becomes larger with smaller interstation distance. The effect reduces quickly with increasing $R$, as seen from the magenta curve in Fig. 5(b).

For a combination of far- and near-field sources, no correction is necessary. Fig. 5 shows that this holds even in the presence of attenuation. $a$ denotes the attenuation factor which reduces the signal amplitude by $\exp (-a \Delta)$, where $\Delta$ is the distance between source and receiver. snr is the signal-to-noise ratio, defined by taking the time-domain cross-correlation and comparing the average absolute amplitude in the signal window to the amplitude in the trailing noise window. An attenuation value of $a=0.005$ could be realistically found at around $5 \mathrm{~s}$ (e.g. Prieto et al. 2009). In all our tests, geometrical spreading is also modelled. Attenuation only shows an effect on the phase velocity in combination with random noise. Otherwise, whitening the contributions from each individual source would cancel out any attenuation effect. Our tests show that for very high attenuation values far sources get damped enough to see some near-field effect. In standard situations we recover the dispersion curve reasonably well. Weemstra et al. (2014) show that for simultaneously acting sources there is no effect from attenuation on the phase-velocity estimation.

We infer that only when near-field signal is dominant, and a reasonable guess of the average distance $R$ can be made, it is worth comparing the fit to a corrected function $f(x)$ (eq. 29). Such a near-field correction might be necessary in the case of island stations, where the main noise caused by the surge is very close and where a good approximation of $R$ can be made.

\subsection{Non-uniform illumination pattern}

Ambient-noise imaging relies on the assumption that sources of ambient noise be uniformly distributed with respect to azimuth $(A$ being independent of $\theta$ as in eq. 6). In real-world applications, this can only be approximately true. The influence of a non-uniform illumination pattern is shown in Fig. 6. In this example, the intensity of the sources changes with azimuth. The distribution roughly resembles the average source intensities for central Europe, with more sources in the North, associated with the Atlantic Ocean. Details on the determination of the azimuthal dependent source intensity in Europe are given in the following section. The changing number of noise sources causes deviations from the spatially uniform velocity of $3.8 \mathrm{~km} \mathrm{~s}^{-1}$. Depending on the orientation of the station pair, the errors are around $\pm 0.02 \mathrm{~km} \mathrm{~s}-1$ at $30 \mathrm{~s}$, corresponding to about 0.5 per cent (Fig. 6c). The behaviour is complex and the relation between the source distribution and the velocity bias is non-linear, changing with period and azimuth of the station couple (see also Yang \& Ritzwoller 2008; Tsai 2009; Weaver et al. 2009; Yao \& Van Der Hilst 2009). Averaging over all station pairs gives, however, the velocity of the initial model. This well-known fact is used in the SPAC method, where no homogeneous distribution of noise sources is needed because of the averaging over different station-station azimuths (Aki 1957; Tsai \& Moschetti 2010). As explained above, only the symmetric part of the cross-correlation spectrum is considered (i.e. the imaginary part is discarded). Therefore, the velocity deviations in Fig. 6(c) are symmetric for a station couple orientation $\pm 180^{\circ}$.

The influence of a non-uniform illumination pattern gets smaller at high frequencies and large interstation distance (Weaver et al. 2009; Yao \& Van Der Hilst 2009), which can be understood when looking at eq. (12), showing that the area of noise sources contributing to the measurement reduces in this case. If only few sources in a very narrow area contribute, the variability in source density is negligible. With 
(a)

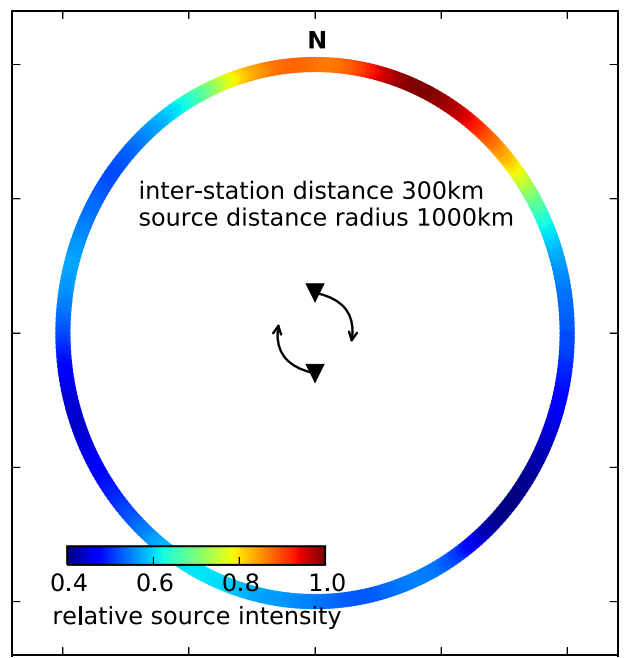

(b)

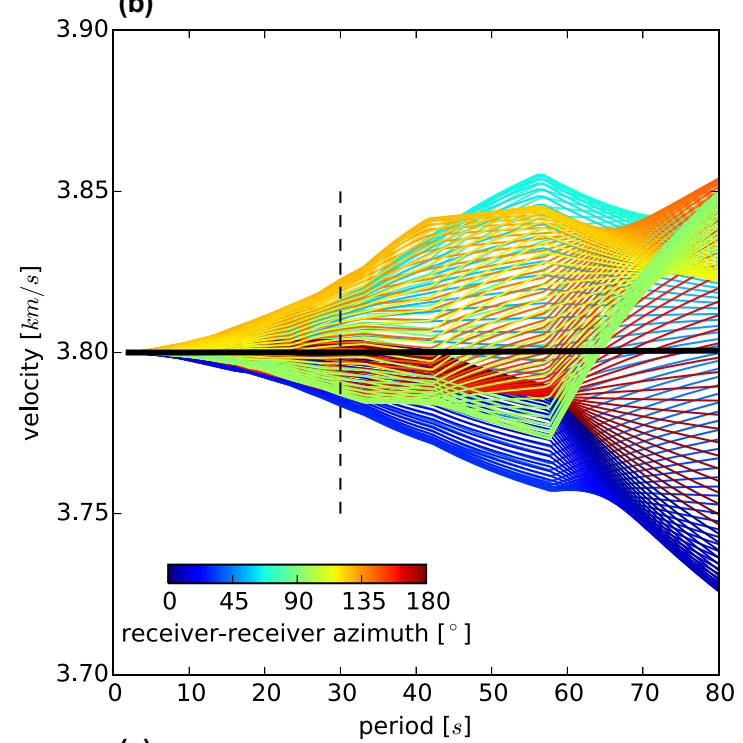

(c)

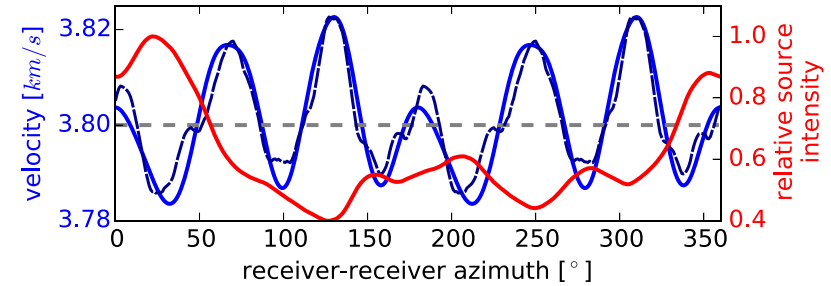

Figure 6. Synthetic tests with non-uniform illumination pattern. (a) Map view of station and source positions. The source intensity follows the average noise-illumination pattern at $30 \mathrm{~s}$ for central Europe; details on the determination are given in the following section. All angles in this figure are given in the geographic coordinate system, that is, an azimuth of zero represents the $\mathrm{N}-\mathrm{S}$ direction. The station couple is rotated in one degree steps. (b) Phase-velocity curves for different station-couple orientations (colours) for the source distribution given in (a). The thick black line is the average of all different azimuth curves. (c) Phase velocity (blue) measured at $30 \mathrm{~s}$ [dashed black line in (b)] depending on the station-station azimuth. The isotropic, constant velocity model is shown as grey dashed line. The blue dashed line gives an approximation of the expected velocity bias according to Weaver et al. (2009). The intensity of the illumination pattern is shown as red curve.

increasing period (or smaller interstation spacing), the Fresnel area gets larger and the effect of source distribution becomes more important, causing larger deviations from the medium velocity. This explains the behaviour seen in Fig. 6(b), where the variability gets larger with increasing period.

Weaver et al. (2009) show that the velocity bias from a non-uniform source illumination is approximately proportional to $\frac{B^{\prime \prime}(\theta)}{B(\theta)}$, where $B(\theta)$ is the source intensity in dependence of the angle $\theta$ and $B^{\prime \prime}(\theta)$ is its second derivative with respect to $\theta$. In Fig. 6(c), we show that the approximation fits well the general pattern of the velocity bias. The fit becomes better towards shorter periods (not shown here). This is due to 
(a)

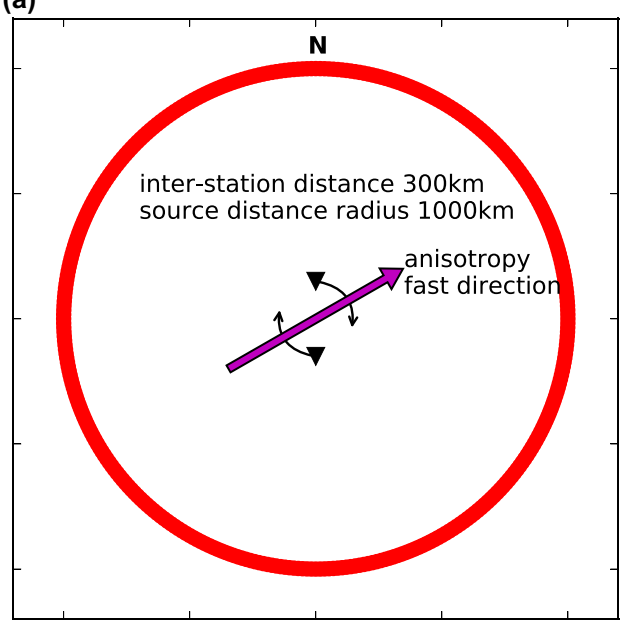

(b)

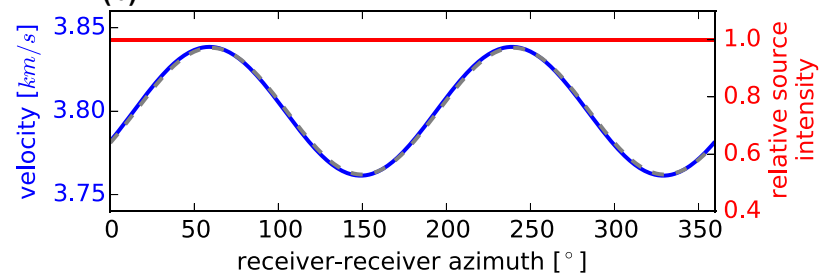

(c)

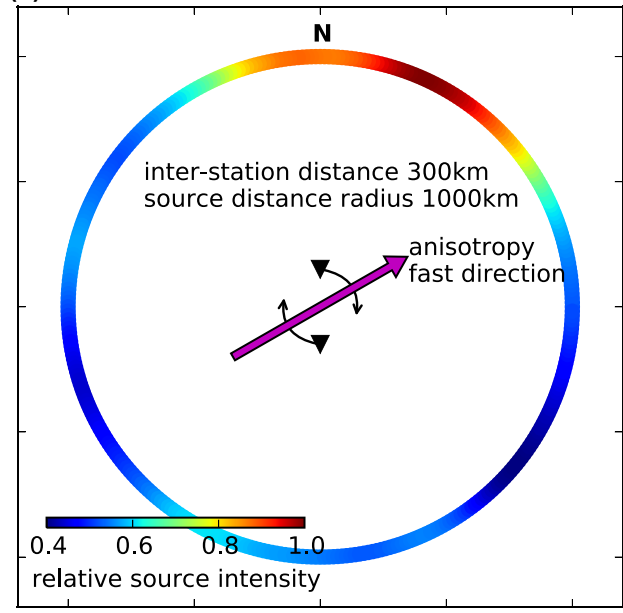

(d)

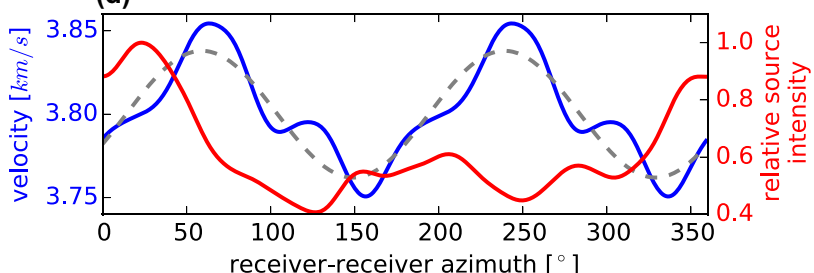

Figure 7. Synthetic model with laterally constant surface wave azimuthal anisotropy. (a) The fast axis of the azimuthal anisotropic medium is at $60^{\circ}$ with 1 per cent higher phase velocity compared to the reference velocity of $3.8 \mathrm{~km} \mathrm{~s}^{-1}$. The slow axis is perpendicular and is 1 per cent slower. (b) Measured phase velocity (blue line) and input medium velocity (grey dashed line), assuming a homogeneous source distribution (red line). The method is able to reproduce the correct velocities in an azimuthally anisotropic velocity model. (c) Same as (a) but with non-homogeneous source distribution, equal to Fig. 6 . (d) The resulting phase velocity is a superposition of the influence of anisotropy (b) and source distribution (Fig. 6c).

the high-frequency approximation applied by Weaver et al. (2009). Note that in Fig. 6(c) we use a symmetric version of the formula of Weaver et al. (2009), given by $\frac{1}{2}\left[\frac{B^{\prime \prime}(\theta)}{B(\theta)}+\frac{B^{\prime \prime}(\theta+\pi)}{B(\theta+\pi)}\right]$. This way we account for the fact that in the presented method we only consider the symmetric part of the cross-correlation spectrum.

\subsection{Azimuthal anisotropy of surface-wave velocity}

In Fig. 7, the influence of an anisotropic surface-wave velocity model is shown, with 1 per cent velocity increase on the fast axis and a 1 per cent decrease on the slow axis. The fast direction is indicated in Fig. 7(a). Recovered phase velocities are exactly the same as in the model. This apparently contradicts the calculations of Yao \& Van Der Hilst (2009), who expect a negative velocity bias in this situation. However, this is explained by their assumption of plane waves, as opposed to our derivation in terms of point sources. As this difference in method concerns the calculation of the synthetic data, we expect no discrepancy in applications to real data for azimuthal anisotropy.

The velocity curve in Fig. 7(d) shows the effect of anisotropic velocities in combination with a non-homogeneous source distribution. The same velocity curve can be obtained by summing the effects of anisotropy and non-homogeneous source distribution separately from the corresponding curves in Fig. 6(c) and Fig. 7.

We infer that azimuthal anisotropy can be recovered without bias by the ANM-at least with the straight ray assumption in this work. The results might be slightly different when ray bending is allowed or finite frequency kernels are used.

\section{COMPARISON BETWEEN AMBIENT-NOISE AND EARTHQUAKE DATA}

The 'Two-Station Method' for measuring surface-wave phase velocity (e.g. Sato 1955; Kovach 1978; Meier et al. 2004; Soomro et al. 2016) is based on using earthquakes as signal sources. When the epicentre is approximately aligned with the station couple, the phase term of the cross-correlation can be evaluated and gives an estimate of the phase velocity. However, selection of smooth parts of the dispersion curves and averaging over a large number of events is necessary to obtain a reliable path-average phase velocity (de Vos et al. 2013; Soomro et al. 2016). In this section, the phase-velocity measurements from the ANM described above are compared to those obtained from the two-station or 'earthquake' method (EQM). Since the parameters of interest are the same as well as the wave types (Rayleigh and Love), the methods are supposed to give coherent results. However, the signal sources are completely independent, although some scattered earthquake coda waves are certainly making a minor contribution to the ambient-noise wavefield. The difference in approach leads to different, method-specific problems. The EQM has to cope with the influence of strong heterogeneity between the stations and on the path from the epicentre to the 

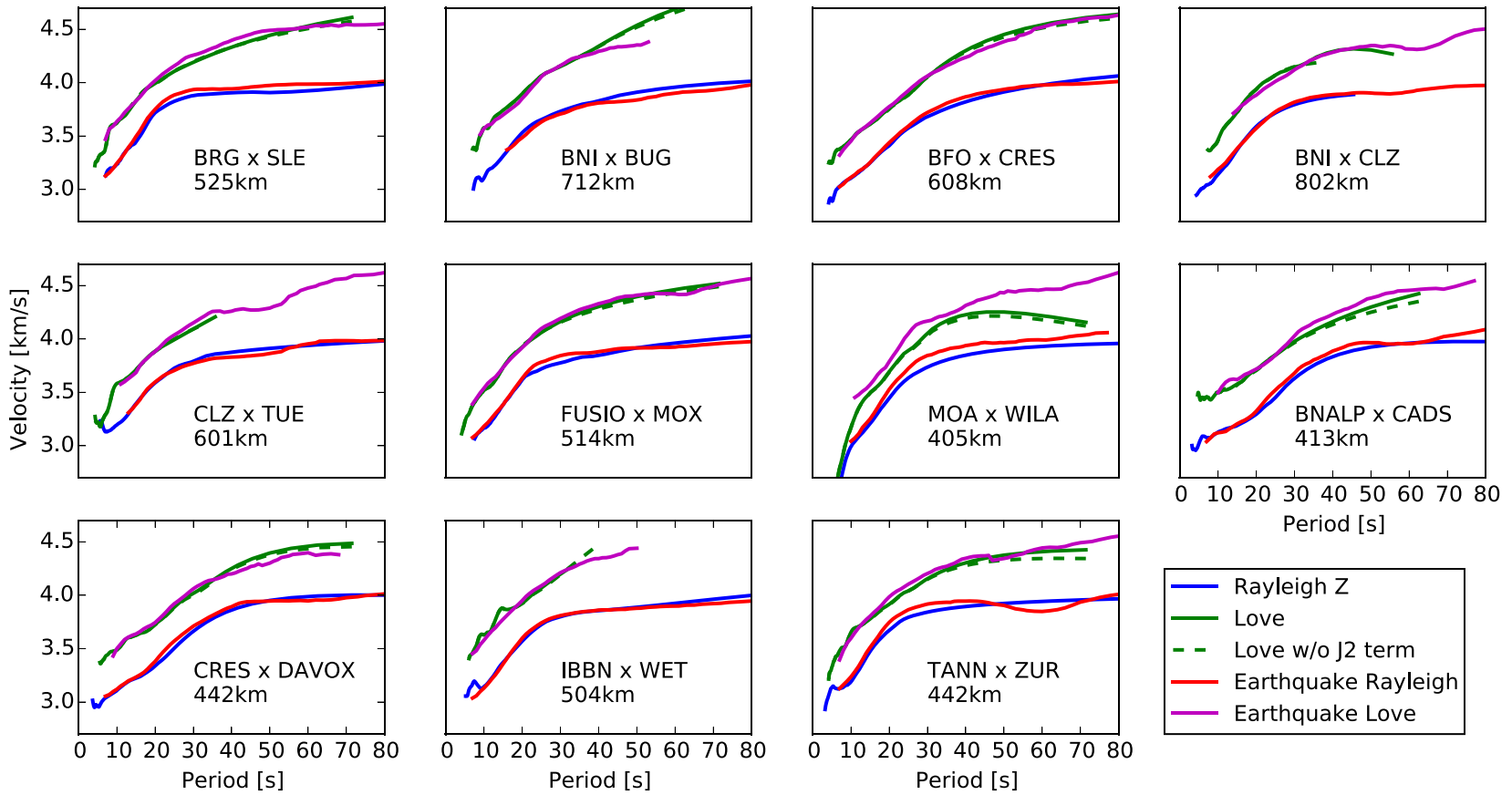

Figure 8. Rayleigh- and Love-wave dispersion curves for different station couples. Each plot gives the phase velocities measured with ambient-noise and earthquake data. For Love waves, the curve is shown with and without correction from eq. (19). Station names and interstation distance are indicated in each window.

stations, the influence of higher modes and noise. It has been shown that time-frequency analysis of the cross-correlation, averaging and selection of smooth parts of single event measurements diminish these effects strongly. However, especially at shorter interstation distances a small positive bias may remain (Soomro et al. 2016). The ANM hinges on the assumption of a perfect distribution of sources and scatterers that have to be uncorrelated and suffers from the low energy content of the coherent part of the wavefield.

The presented data were recorded in central Europe, mostly the Alpine region and are publicly available. The ambient-noise data is prepared and evaluated according to the method presented above. Details on the compilation of the earthquake-based data set based on an automated processing scheme involving a number of quality checks are given in Soomro et al. (2016).

\subsection{Dispersion curves}

In Fig. 8, we compare phase-velocity dispersion curves as measured via EQM versus ANM for several station couples. The phase velocity has been picked using the averaged EQM as reference in order to introduce no bias (see also Fig. 4). We have not noticed any dependence of the results on the reference curve as long as it does not deviate more than $\pm 1 \mathrm{~km} \mathrm{~s}^{-1}$ from the expected value. Both Love and Rayleigh wave phase velocities agree well, with discrepancies generally well below $0.1 \mathrm{~km} \mathrm{~s}^{-1}$. The effects from the Love wave correction from eq. (19), that is, including the $J_{2}$ term, are shown (see also Fig. 3). The difference is largest at long period and short interstation distance, and becomes approximately negligible below $40 \mathrm{~s}$. Not all measurements are of equal quality: just one or two wrong picks due to noise at the low-frequency end of the cross-correlation spectrum can lead to important deviations such as for station couple MOA-WILA.

Fig. 9 provides a more general comparison by showing the averaged measurements from all station pairs for which data from both methods are available. The average discrepancies are as large as almost $0.05 \mathrm{~km} \mathrm{~s}^{-1}$ for Rayleigh and $0.06 \mathrm{~km} \mathrm{~s}^{-1}$ for Love waves; in both cases this amounts to approx. 1 per cent of the reference velocity. Fewer Love-wave dispersion measurements are available which could cause the slightly higher discrepancies seen in Fig. 9(b). Apart from the dispersion curves, also the data coverage for the ANM depending on the period is shown. For this comparison, we choose only station pairs where measurements at all sampled periods between 20 and $50 \mathrm{~s}$ are available. The Love-wave discrepancy is reduced, on average, by accounting for the $J_{2}$ term in Love-wave cross-correlation (Section 2.4 ). The effect of the $J_{2}$ term is also shown for the R-component dispersion curve. However, this correction alone cannot explain the discrepancies between the methods.

The differences between ANM and EQM are highest between 25 and $35 \mathrm{~s}$. The histogram in Fig. 9(d) shows how the differences between the two methods are distributed at $25 \mathrm{~s}$ period. Few outliers can be identified for the Rayleigh-wave comparison, but they are not numerous enough to contribute significantly to this discrepancy.

Average faster velocities from the EQM in comparison to the ANM have also been reported by Zhou et al. (2012) (0.012 $\mathrm{km} \mathrm{s}^{-1}$ from comparison of phase-velocity maps), Ritzwoller et al. (2011) (0.004 $\mathrm{km} \mathrm{s}^{-1}$ from comparison of phase-velocity maps), Shen et al. (2013) $\left(0.001 \mathrm{~km} \mathrm{~s}^{-1}\right.$ from comparison of phase-velocity maps) and Yao et al. (2008) $\left(0.06 \mathrm{~km} \mathrm{~s}^{-1}\right.$ from comparison of averaged phase velocity 
(a)

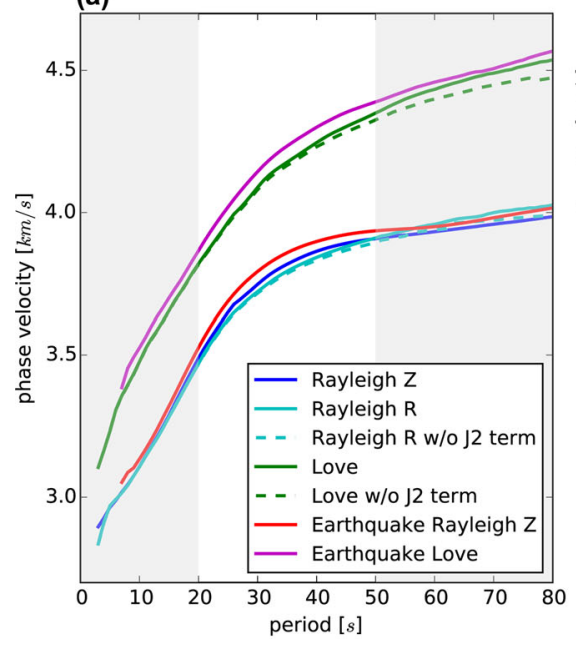

(b)

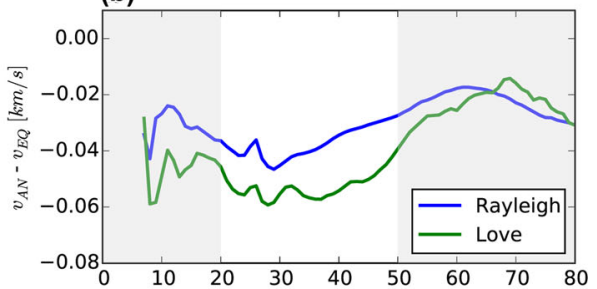

(c)

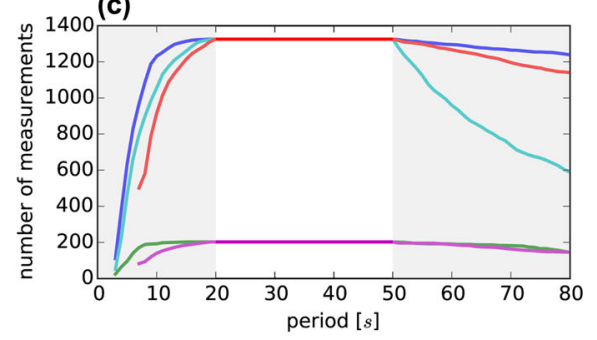

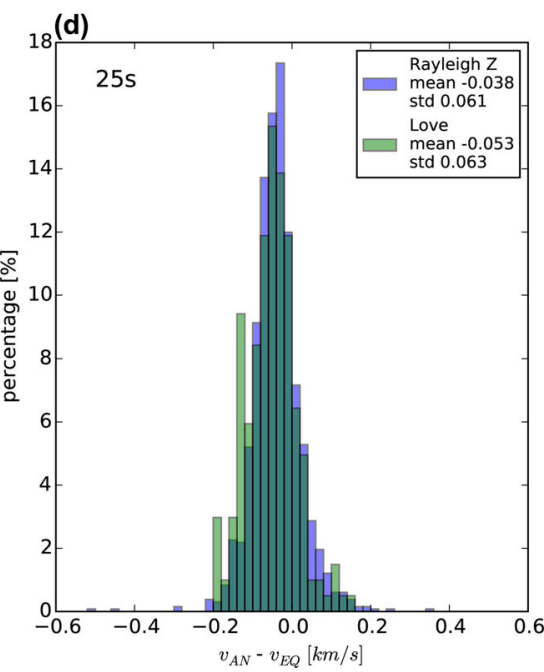

Figure 9. (a) Average of the phase-velocity dispersion curves, comparing EQM and ANM. The ANM covers periods down to about $4 \mathrm{~s}$ whereas the EQM allows measurements up to very long periods of $250 \mathrm{~s}$ and beyond. The same station couples are compared. The valid range for comparison is distinguished by a white background. Noise measurements predict slightly slower velocities, with differences around $0.04-0.06 \mathrm{~km} \mathrm{~s}^{-1}$. The dispersion curves for Love and Rayleigh R-component correlations are shown with (solid line) and without (dashed line) the additional J2-term of eq. (19). (b) Difference between average dispersion curves from (a). (c) Number of data points used in the comparison. Only station pairs where data between 20 and $50 \mathrm{~s}$ are available are used. Fewer Love-wave measurements are available for the comparison. Line colours correspond to the legend in (a). (d) Differences between EQ and AN measurements at $25 \mathrm{~s}$ period. Their distribution is approximately Gaussian, with few outliers.

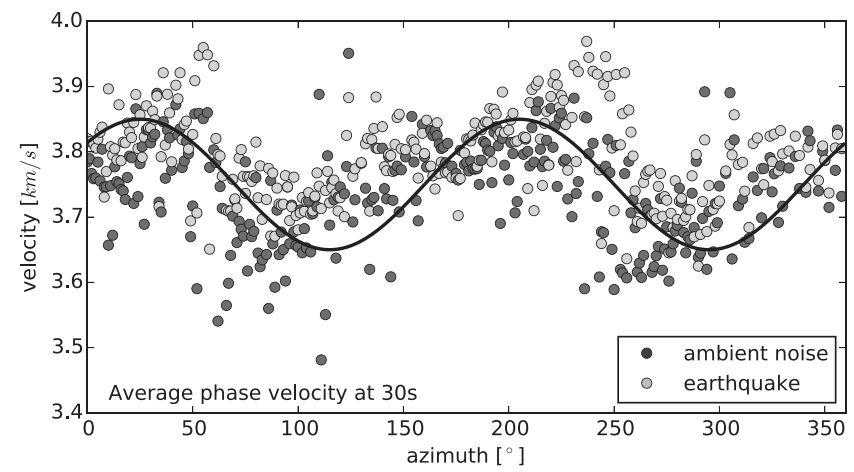

Figure 10. Rayleigh phase velocity at $30 \mathrm{~s}$ period for different receiver-receiver azimuth; averaged over all station couples with identical orientation. The ANM and the EQM show the same pattern of fast and slow direction, with fast direction around $25^{\circ}$ and $205^{\circ}$ and $\pm 0.1 \mathrm{~km} \mathrm{~s}^{-1}$ variability as shown by the black line. The average discrepancy of $0.05 \mathrm{~km} \mathrm{~s}^{-1}$ between the earthquake and the ambient-noise measurements is well visible.

curves) - all taken around $30 \mathrm{~s}$. Without stating whether the discrepancy is positive or negative, Köhler et al. (2012) also find residuals of up to 1.5 per cent at $25 \mathrm{~s}$. To our knowledge, there are no studies that show faster measurements from the ANM, which leads us to believe that this discrepancy is systematic. There are several possible reasons for the bias between ambient-noise and earthquake results:

(i) In Fig. 5, we show that near-field sources represent a possible reason for erroneous phase-velocity measurements from ambient noise. However, we can infer from Fig. 9 that our ANM measurements are not biased by the (a priori possible) presence of many near-field sources: this would have resulted in ANM estimates of velocity being higher than EQM ones, contradicting the data in Fig. 9. This is in agreement with the fact that most ambient-noise signal recorded in Europe is generated in the surrounding seas/oceans.

(ii) Non uniformity in noise-source distribution with respect to azimuth. This influence is hard to quantify as the effects of a non-uniform illumination pattern are complex and can cause positive as well as negative velocity deviations (e.g. Yang \& Ritzwoller 2008; Tsai 2009; Yao \& Van Der Hilst 2009). However, this should cancel out in the averaging procedure applied in Fig. 9, as shown in Section 3.2.

(iii) Off-path propagation between the earthquake event and the station couple. The EQM uses the difference in epicentral distances and not the interstation distance to calculate phase differences, which reduces the effect from earthquakes which are not located exactly on the station great circle. The bias from event mislocation would be frequency independent. Therefore, event mislocation is an unlikely cause for the discrepancies evidenced in Figs 9 and 11. Velocity heterogeneity causes deviations from the direct path and introduces an error in the differential path. For short interstation distances, this will result in a positive bias of the estimated phase velocities. At larger interstation distances this effect is however reduced by wave front healing and by averaging over events from different source regions (Soomro et al. 2016), because earth heterogeneity additionally curves wave fronts, which can result in both positive and negative perturbations of the phase velocity (Wielandt 1993). 

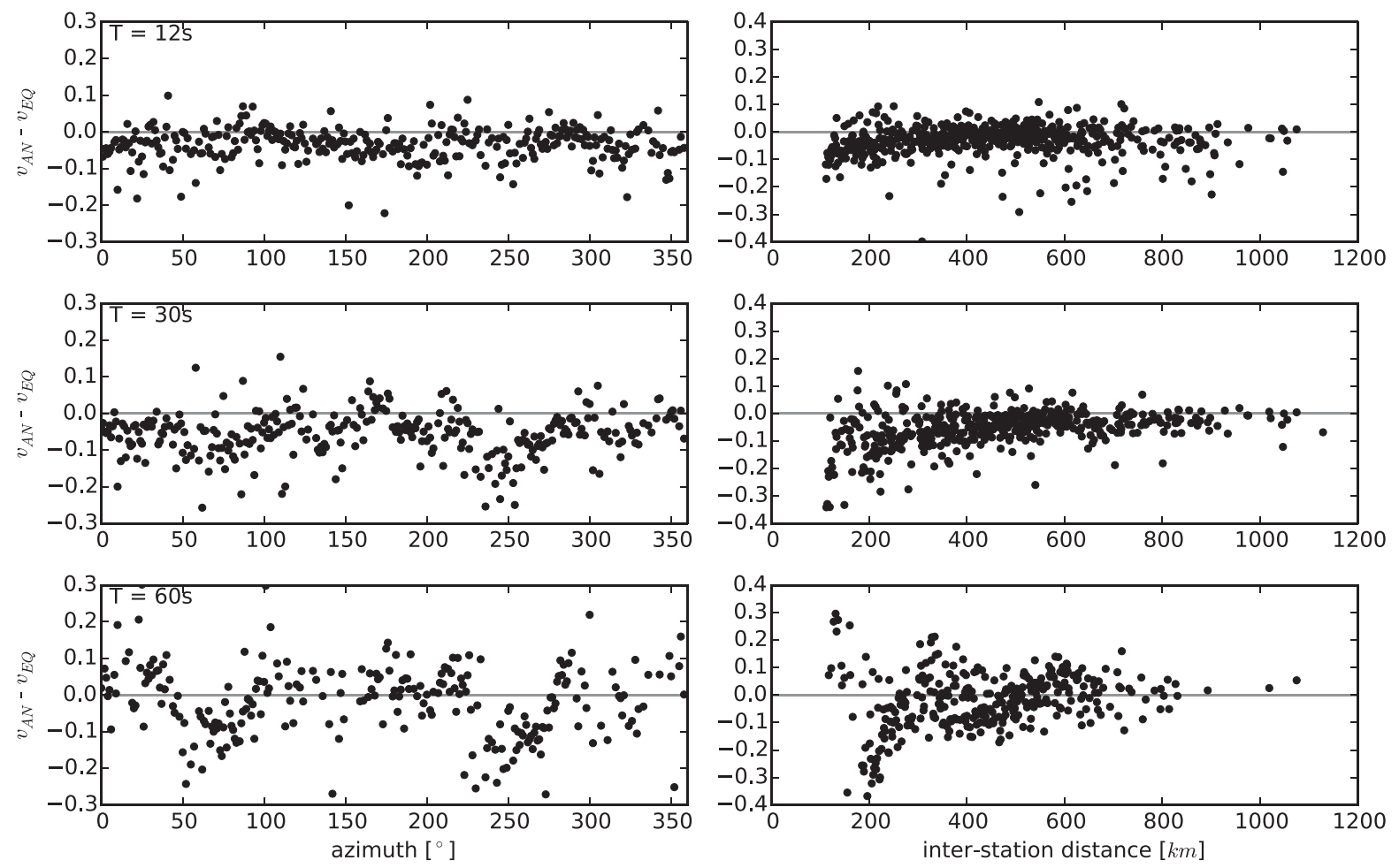

Figure 11. Difference in the Rayleigh-wave phase velocity from earthquake $\left(v_{\mathrm{EQ}}\right)$ and ambient-noise $\left(v_{\mathrm{AN}}\right)$ measurements in km $\mathrm{s}^{-1}$. The differences are an average of all station couples sorted according to the azimuth of the station couple orientation (left) and interstation distance (right) at three different periods. An azimuth of zero stands for a north-south orientation. The variability gets higher with longer periods, which is because of the decrease in number of measurements but also because of the larger Fresnel zone. The Fresnel zone effect is also visible in the variation with distance, getting smaller for large interstation distances.

(iv) Teleseismic, single-event measurements will have a broader sensitivity kernel. This effect is diminished by averaging (de Vos et al. 2013). In general, the sensitivity to structure will be slightly different for the ANM and the EQM, which can give different velocity estimations when the medium velocity is laterally changing.

(v) Soomro et al. (2016) mention the influence of surface-wave overtones as another possible cause for a positive bias in the EQM estimates of phase velocity. This effect is minimized by the time-frequency analysis. No fk-filter or time window selection is done in case of the ambientnoise data. The relative contribution of higher modes to the ambient-noise wavefield are estimated quite differently in the literature and seem to be dependent of the region. However, most authors state that fundamental-mode surface waves dominate the ambient-noise records, especially at periods longer than $5 \mathrm{~s}$ (Lacoss et al. 1969; Pedersen \& Krüger 2007; Nishida et al. 2008; Yao et al. 2011). Some preliminary numerical tests of our method indicate that even in the presence of a secondary, faster signal emitted by each noise source, roughly corresponding to the first higher mode, the fundamental mode phase-velocity signal dominates the phase-velocity measurement procedure and the fundamental mode phase velocity is correctly reconstructed. Finally, it should be noted that misidentification of fundamental-mode versus overtone phases would result in an overestimate (rather than the observed, suspected underestimate) of fundamental mode phase velocity. We infer that higher mode contamination in the ambient-noise measurements is unlikely to explain the observed EQM-ANM discrepancy.

(vi) In principle, scattering may introduce spurious correlations and therefore influence the measured velocity (e.g. Boschi et al. 2013). However, on the scale considered here, it is reasonable to assume that many scattering structures are present and that their geographic distribution is relatively uniform. In such a situation, it has been shown (Larose et al. 2008) that scatterers will actually contribute to a better reconstruction of the Green's function. If present, scattering might have some systematic effects on EQM data, but these are presumably small and their analysis is beyond the scope of this study.

We show in Fig. 10 phase-velocity estimates from both ANM and EQM at $30 \mathrm{~s}$ period, for all possible station couples, as a function of receiver-receiver azimuth. The discrepancy between ANM and EQM does not show any strong dependence on azimuth, indicating that, if the European crust/upper mantle is azimuthally anisotropic, both methods should resolve this equally well. Indeed, Fig. 10 does suggest that the region of interest is anisotropic. Fry et al. (2010) show that, at $30 \mathrm{~s}$, the average anisotropy fast direction in the western and central Alpine region is around $0^{\circ}$ with 2.5 per cent anisotropic anomaly. The magnitude fits our results very well, with 2.5 per cent of $3.8 \mathrm{~km} \mathrm{~s}^{-1}$ being around $0.1 \mathrm{~km} \mathrm{~s}^{-1}$. The direction deviates slightly from the one shown in Fig. 10, but this is probably because we average over a larger region. 

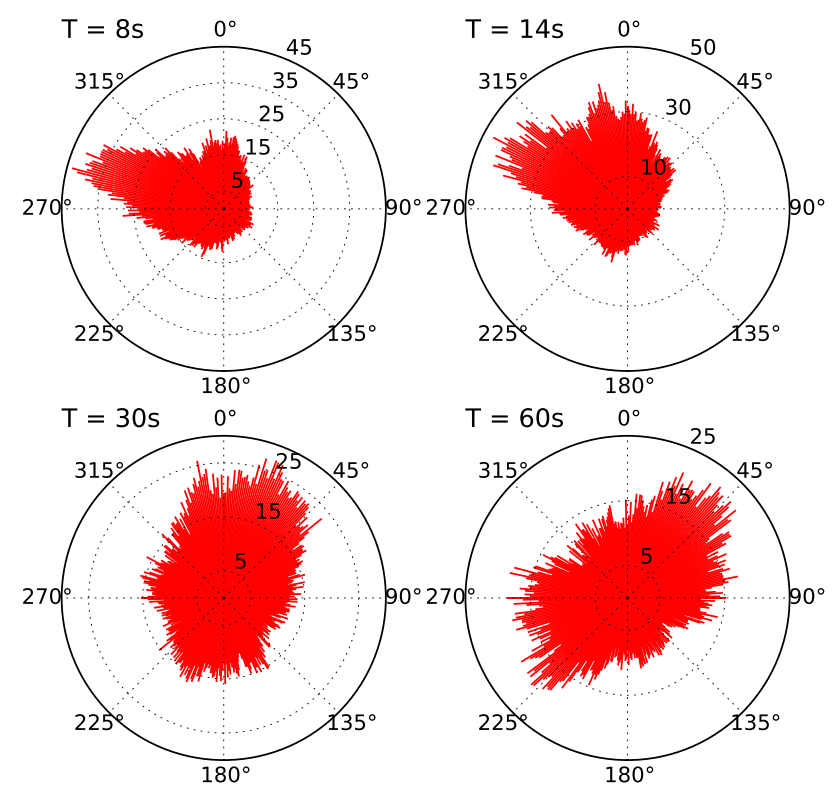

Figure 12. Signal-to-noise ratio at different periods according to the azimuth. A high signal-to-noise ratio shows strong sources generating ambient noise coming from the specific direction. The periods at 8 and $14 \mathrm{~s}$ stand for the primary and secondary microseisms band, respectively. In both cases, the strongest source activity comes from the Atlantic. The long-period sources are distributed more isotropically. The distribution at $30 \mathrm{~s}$ serves as model for the synthetic test in Fig. 7.

Fig. 11 separates the effects from station couple orientation, interstation distance and period. The effect of the sensitivity kernels can be recognized easily: the variability of the noise data increases as the station-station spacing gets smaller and with increasing period. This effect could be explained with a non-uniform illumination pattern as indicated above (Fig. 7).

The fluctuations with azimuth are most likely not associated with anisotropy, since that seems to be resolved by both ANM and EQM (Fig. 10). In the shown case, the average velocity discrepancies are as high as 2.5 percent for certain orientations. The difference decreases for longer interstation distances. This might be partly caused by a small remaining positive bias in the EQM for small interstation distances.

\subsection{Distribution of noise sources}

Fig. 12 shows the signal-to-noise ratio for Rayleigh waves at different periods, obtained by filtering the stacked cross-correlations and comparing the maximum signal amplitude with the trailing noise in the time domain, according to Yang \& Ritzwoller (2008). The bounds of the signal window are given by the interstation distance divided by the maximum $\left(5 \mathrm{~km} \mathrm{~s}^{-1}\right)$ and minimum $\left(2 \mathrm{~km} \mathrm{~s}{ }^{-1}\right)$ expected velocity, respectively. We thus obtain a signal-to-noise estimate for each station couple for the forward (waves travelling from station 1 to 2 ) and backwards (vice-versa) empirical Green's function. In the first and secondary microseisms band, a preferential direction of noise sources is clearly visible with most of the energy coming from the Atlantic (Pedersen \& Krüger 2007; Campillo \& Roux 2015).

The illumination pattern used to construct synthetic data in Section 3 (Fig. 7) was derived from the signal-to-noise distribution at $30 \mathrm{~s}$ shown in Fig. 12. We already demonstrated that such noise source distribution does not cause any systematic bias. Further synthetic tests of ANM, focused on the effects of non-uniform station distribution (results not shown here in the interest of brevity) did not result in systematic errors, either. This suggests that the actual station distribution covers sufficiently well the whole azimuthal range. The synthetic tests, however, significantly simplify the illumination pattern and the medium. For example, the source intensity distribution will change for every station pair considered, so that the zero-average bias, as seen in the synthetic test, might not hold. A non-uniform velocity model causes also deviations from the ray-theoretical measurement between two stations (e.g. Tsai 2009). The expected effects are, however, small for a sufficiently large number of stations considered.

\subsection{Phase-velocity maps}

The analysis of Section 4 suggests that a small, but possibly significant discrepancy exists between phase-velocity estimates based on the ANM and the EQM. To quantify its practical relevance, we next determine phase-velocity maps from both ANM and EQM data and evaluate their agreement (Fig. 13). The phase velocities at a period of $25 \mathrm{~s}$ are taken as an intermediate value where both approaches have good data coverage. In the inversion process. the test region is divided in $0.1^{\circ}$ wide squares and the sensitivities are calculated in ray-theory approximation, following, for example, Verbeke et al. (2012). Both ANM and EQM inversions are parametrized and regularized in the same way, via roughness damping only (e.g. Boschi \& Dziewonski 1999). Importantly, we only include in this exercise station couples for which data from both methods are available at the period in question. 
(a)

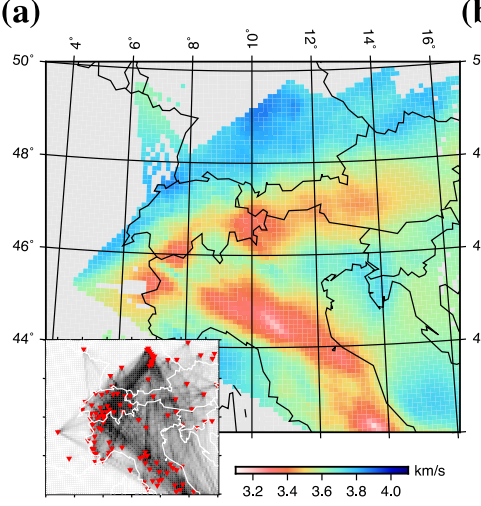

(b)

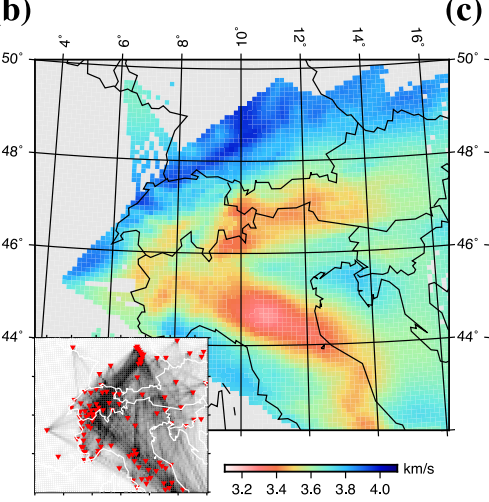

(c)

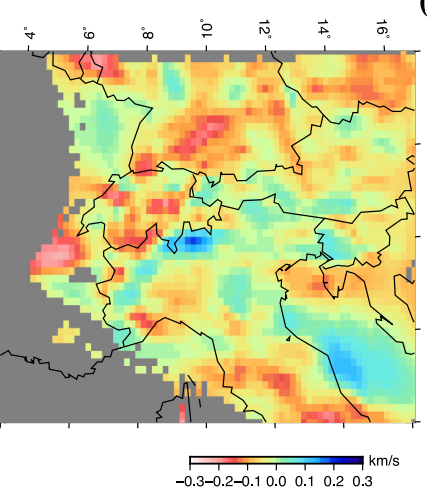

(g)

(e)

(f)
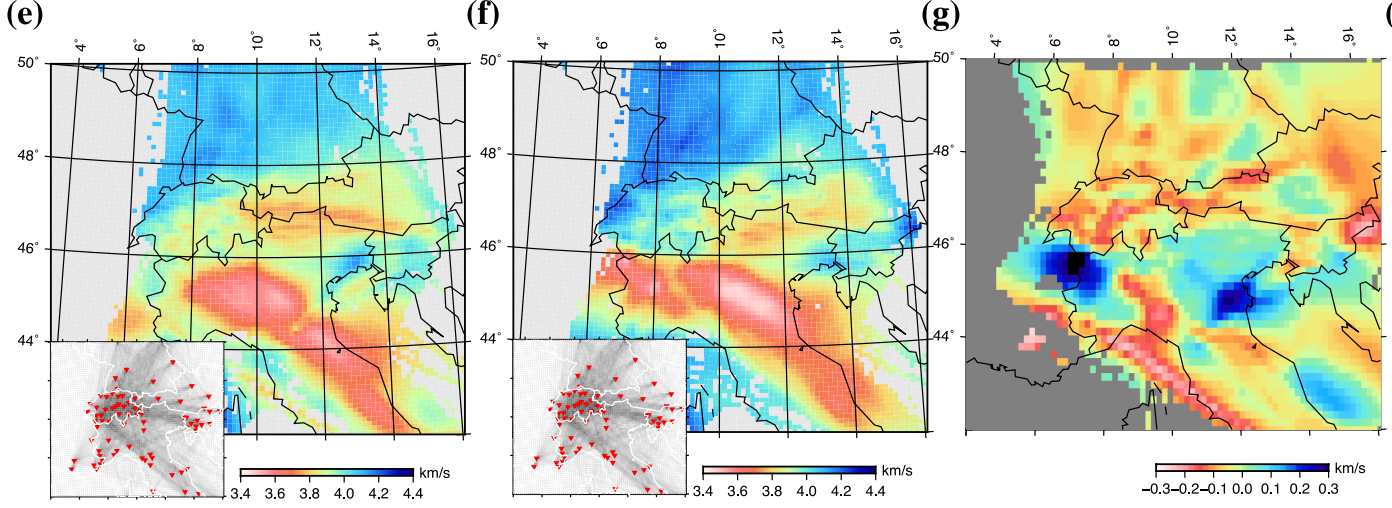

(d)

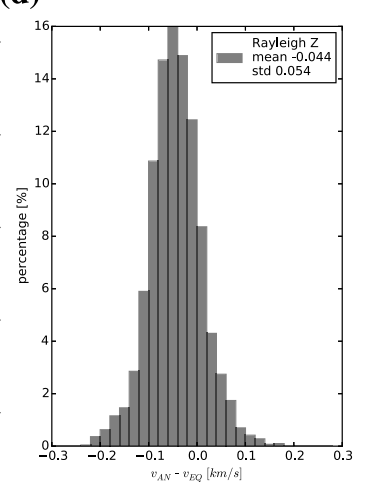

(h)

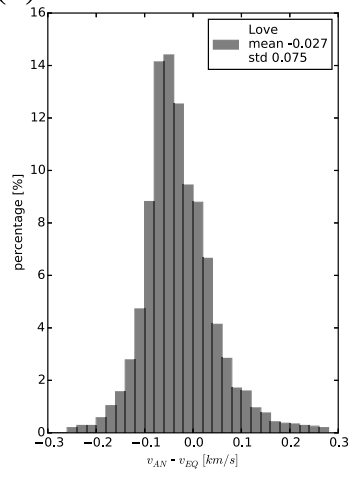

Figure 13. Comparison of phase-velocity maps at 25 s period for Rayleigh (top) and Love (bottom) waves. (a) Rayleigh, ANM, (b) Rayleigh, EQM, (c) difference between (a) and (b). (d) Differences from (c) as histogram. (e-h) Same as (a-d) for Love waves.

The Rayleigh-wave maps (Fig. 13) should be seen as a depth-average of the lower-crust/upper mantle velocity structures. Both ANM and EQM show the thickened crust underneath the Alpine orogen, corresponding to low phase velocities. Pronounced velocity reductions characterize also the Po-plain basin in northern Italy and the Apennines. The overall structures of Fig. 13 are in good agreement with phase-velocity maps from Stehly et al. (2009); Verbeke et al. (2012); Molinari et al. (2012).

Differences between ANM and EQM are in the range of $\pm 0.3 \mathrm{~km} \mathrm{~s}^{-1}$ for both Love- and Rayleigh waves. The inversion and smoothing process suppresses outliers that are visible in $9 \mathrm{~d}$. Otherwise, discrepancies in the phase-velocity maps are comparable to the ones from direct comparison of the phase-velocity curves.

Several studies similarly compare Rayleigh phase-velocity maps: Zhou et al. (2012) report maximum phase-velocity differences of 0.2 $\mathrm{km} \mathrm{s}^{-1}$, but with a smaller mean of $-0.012 \mathrm{~km} \mathrm{~s}^{-1}$. Yang \& Ritzwoller (2008) find deviations of $0.15 \mathrm{~km} \mathrm{~s}^{-1}$. Very small discrepancies are found in the maps of Shen et al. (2013) who give a maximum of $0.08 \mathrm{~km} \mathrm{~s}^{-1}$ and a mean of $-0.001 \mathrm{~km} \mathrm{~s}^{-1}$. This is similar to the small mean deviation of $-0.004 \mathrm{~km} \mathrm{~s}^{-1}$ reported by Ritzwoller et al. (2011).

Phase-velocity curves from the EQM are determined for each event aligned with a station pair, and then averaged over all such events. This results in smoother estimates of the phase-velocity dispersion. Additionally, (Soomro et al. 2016) applied certain smoothness constraints, discarding parts of the dispersion curve that are particularly rough. We do not smoothen our phase-velocity curves and some roughness remains as seen in Fig. 4. This results in higher standard deviations and higher/lower maximum/minimum values of $c$. We have seen that an inhomogeneous source distribution influences the measured phase velocity for individual station couples: we show above that this does not influence the mean, but it could cause a higher standard deviation.

Love waves at a given period are sensitive to shallower structures than Rayleigh waves at the same period. The Love-wave maps of Fig. 13 show similar structures as the Rayleigh-wave ones. Higher sensitivities to shallower crustal structures result in a very prominent low-velocity zone associated with the Po-plain. The mean discrepancy gets smaller in the phase-velocity map comparison, compared to the phase-dispersion curves (Fig. 9). This is presumably explained by the geographic distribution of station-station phase-velocity measurements on the map and by the smoothing in the inversion process. To our knowledge, no other studies compare the results of ANM and EQM for Love waves. Our results suggest that the bias between the methods is independent of the wave type.

We evaluate the size of the structures that are resolvable by the station configuration of Fig. 13 with a checkerboard test in Fig. 14. Theoretical receiver-receiver dispersion curves corresponding to this synthetic model are computed using ray-theory (but neglecting ray bending) and a finer parametrization $(1 \mathrm{~km})$ than that of the tomographic inversions. They are then inverted with the same parametrization and regularization parameters as on the maps above. The comparison shows that high discrepancies in Fig. 13 even occur in regions of good coverage. 
(a)

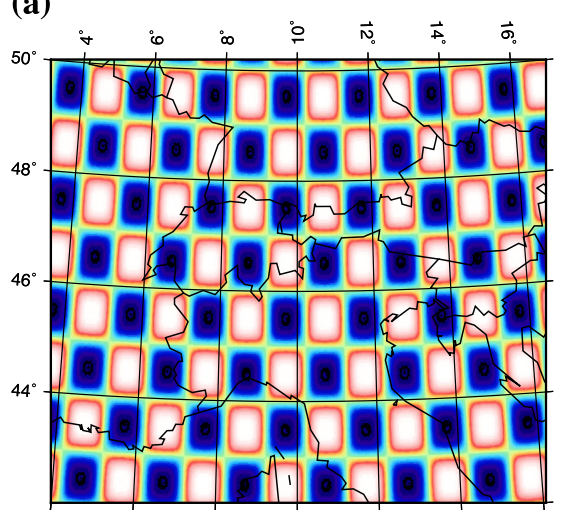

(b)

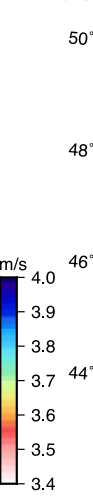

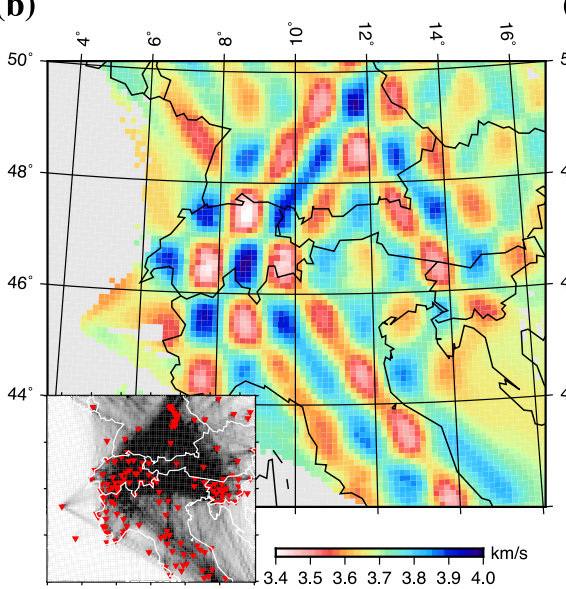

(c)

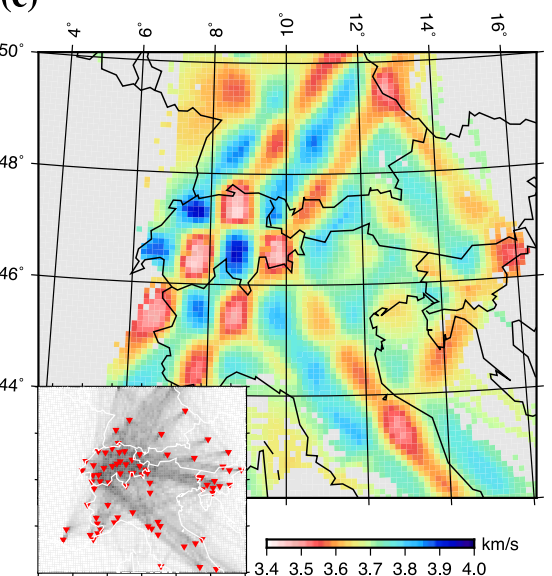

Figure 14. Resolution test with the ray coverage from Fig. 13. (a) Synthetic checkerboard model with $1^{\circ}$ cells. (b) Recovered phase-velocity map for the Rayleigh-wave case. (c) Same as (b) for the Love-wave case.

\section{CONCLUSIONS}

In this work, we prove the validity of the ANM by comparison with the well-established EQM (Sato 1955; Kovach 1978; Meier et al. 2004; Soomro et al. 2016). The basic theory of the ANM is revised in order to evaluate its limitations and the approximations that it involves. This includes a far-field assumption, the effects of anisotropic source distribution and azimuthal velocity anisotropy, whose influence is examined using synthetic tests. We also discuss in detail how Love-wave phase velocity can be extracted from ambient noise, show that the mathematical expression for noise cross-correlation is slightly different for Love waves with respect to the vertical component of Rayleigh waves, and verify that the error caused by neglecting such difference (as has sometimes been done) is, generally, small, but can become important for short interstation distance and long periods. We show that the same issue arises when extracting Rayleigh waves from the radial, rather than vertical component of recorded noise.

The central European Alps are taken as example region to test the differences between the two methods. A good agreement in the resulting phase velocities is found with average discrepancies smaller than $0.06 \mathrm{~km} \mathrm{~s}^{-1}$. ANM-based velocity estimates are, on average, slightly lower than those obtained by the EQM, as indicated also by other authors (Yao et al. 2008; Ritzwoller et al. 2011; Zhou et al. 2012; Shen et al. 2013), reporting average discrepancies for Rayleigh-wave phase velocity of $-0.07,-0.004,-0.012$ and $-0.001 \mathrm{~km} \mathrm{~s}^{-1}$, respectively. The fact that this bias seems to be very stable and not randomly changing with period, distance and/or azimuth suggests that it should be explained by methodological differences. The largest average discrepancy is found for both Love and Rayleigh waves at periods around $30 \mathrm{~s}$, where Rayleigh waves are particularly sensitive to heterogeneous Moho topography (Meier et al. 1997).

We extract the azimuthally dependent signal-to-noise ratio at this period and use it in a synthetic model in order to test for the effects of a non-uniform illumination pattern. Our synthetic results suggest that, on average, the observed bias in noise-source distribution does not affect significantly our ANM phase-velocity estimates. We verify also that azimuthal anisotropy in surface-wave velocity can be reconstructed properly by the ANM. We infer that the small residual discrepancy between EQM and ANM dispersion measurements is the result of a combination of factors, including lateral velocity variations and differences in sensitivity of EQM versus ANM data to structure, which are not accounted for if, like here, the ray-theory approximation is used. We also assume a small influence from higher modes on the EQM. Further research will be necessary to fully explain the bias between the methods.

We finally show that, in the context of applications to crust and upper mantle imaging, the discussed discrepancy is sufficiently small to be negligible with respect to other effects: the good agreement between phase-velocity maps based on EQM versus ANM is illustrated by Fig. 13. This compatibility is key to combining the results of both methods in order to cover a wider period range as done, for example, by Yao et al. (2008) or Zhou et al. (2012). We suggest that joint inversion of ANM and EQM dispersion data will provide 3-D images of the uppermost mantle at resolution significantly higher than achieved by either data set when inverted alone. In this endeavour, it will be important to take into account some complex effects that we quantified here, namely the contribution of $J_{2}$ to the Love-wave and radial-component ANM cross-correlation spectra.

\section{ACKNOWLEDGEMENTS}

We are grateful to Göran Ekström, Jean-Paul Montagner, the editor Michael Ritzwoller and the two anonymous reviewers for their helpful comments. We are grateful to all the network operators providing data to the EIDA archive (http://www.orfeus-eu.org/eida, last accessed April 2015). We thank the makers of Obspy (Beyreuther et al. 2010). Graphics were created with Python Matplotlib and GMT (Wessel et al. 2013). $\mathrm{CW}$ is supported by the Netherlands Research Centre for Integrated Solid Earth Science (ISES). This project has received funding from the European Union's Horizon 2020 research and innovation programme under the Marie Sklodowska-Curie grant agreement No. 641943. 


\section{REFERENCES}

Abramowitz, M. \& Stegun, I.A., 1964. Handbook of Mathematical Functions: With Formulas, Graphs, and Mathematical Tables, no. 55, Courier Corporation.

Agius, M.R. \& Lebedev, S., 2013. Tibetan and Indian lithospheres in the upper mantle beneath Tibet: evidence from broadband surface-wave dispersion, Geochem. Geophys. Geosyst., 14(10), 4260-4281.

Aki, K., 1957. Space and time spectra of stationary stochastic waves, with special reference to microtremors, Bull. Earthq. Res. Inst., 35, 411-451.

Aki, K. \& Richards, P.G., 2002. Quantitative Seismology, University Science Books.

Basini, P., Nissen-Meyer, T., Boschi, L., Casarotti, E., Verbeke, J., Schenk, O. \& Giardini, D., 2013. The influence of nonuniform ambient noise on crustal tomography in Europe, Geochem. Geophys. Geosyst., 14(5), 1471-1492.

Bender, C.M. \& Orszag, S.A., 1978. Advanced Mathematical Methods for Scientists and Engineers, McGraw-Hill.

Bensen, G., Ritzwoller, M., Barmin, M., Levshin, A., Lin, F., Moschetti, M., Shapiro, N. \& Yang, Y., 2007. Processing seismic ambient noise data to obtain reliable broad-band surface wave dispersion measurements, Geophys. J. Int., 169(3), 1239-1260.

Bettig, B., Bard, P., Scherbaum, F., Riepl, J., Cotton, F., Cornou, C. \& Hatzfeld, D., 2001. Analysis of dense array noise measurements using the modified spatial auto-correlation method (SPAC): application to the Grenoble area, Boll. Geofis. Teor. Appl., 42(3-4), 281-304.

Beyreuther, M., Barsch, R., Krischer, L., Megies, T., Behr, Y. \& Wassermann, J., 2010. ObsPy: a Python toolbox for seismology, Seismol. Res. Lett., 81(3), 530-533.

Boschi, L. \& Dziewonski, A.M., 1999. High-and low-resolution images of the earth's mantle: implications of different approaches to tomographic modeling, J. geophys. Res., 104(B11), 25 567-25 594.

Boschi, L. \& Weemstra, C., 2015. Stationary-phase integrals in the cross-correlation of ambient noise, Rev. Geophys., 53(2), 411-451.

Boschi, L., Weemstra, C., Verbeke, J., Ekström, G., Zunino, A. \& Giardini, D., 2013. On measuring surface wave phase velocity from station-station cross-correlation of ambient signal, Geophys. J. Int., 192(1), 346-358.

Campillo, M. \& Roux, P., 2015. Crust and lithospheric structure - Seismic imaging and monitoring with ambient noise correlations, in Treatise on Geophysics, 2nd edn, Vol. 1, pp. 391-417, eds Romanowicz, B. \& Dziewonski, A., Elsevier, Amsterdam.

de Vos, D., Paulssen, H. \& Fichtner, A., 2013. Finite-frequency sensitivity kernels for two-station surface wave measurements, Geophys. J. Int., 194(2), 1042-1049.

Ekström, G., 2014. Love and Rayleigh phase-velocity maps, 5-40 s, of the western and central USA from USArray data, Earth planet. Sci. Lett., 402, 42-49.

Ekström, G., Abers, G.A. \& Webb, S.C., 2009. Determination of surfacewave phase velocities across USArray from noise and Aki's spectral formulation, Geophys. Res. Lett., 36(18), doi:10.1029/2009GL039131.

Endrun, B., Meier, T., Bischoff, M. \& Harjes, H.-P., 2004. Lithospheric structure in the area of Crete constrained by receiver functions and dispersion analysis of Rayleigh phase velocities, Geophys. J. Int., 158(2), 592-608.

Endrun, B., Lebedev, S., Meier, T., Tirel, C. \& Friederich, W., 2011. Complex layered deformation within the Aegean crust and mantle revealed by seismic anisotropy, Nat. Geosci., 4(3), 203-207.

Friedrich, A., Krüger, F. \& Klinge, K., 1998. Ocean-generated microseismic noise located with the Gräfenberg array, J. Seismol., 2(1), 47-64.

Fry, B., Deschamps, F., Kissling, E., Stehly, L. \& Giardini, D., 2010. Layered azimuthal anisotropy of Rayleigh wave phase velocities in the European Alpine lithosphere inferred from ambient noise, Earth planet. Sci. Lett., 297(1), 95-102.

Halliday, D. \& Curtis, A., 2008. Seismic interferometry, surface waves and source distribution, Geophys. J. Int., 175(3), 1067-1087.
Harmon, N., Gerstoft, P., Rychert, C.A., Abers, G.A., Salas de La Cruz, M. \& Fischer, K.M., 2008. Phase velocities from seismic noise using beamforming and cross correlation in Costa Rica and Nicaragua, Geophys. Res. Lett., 35(19), doi:10.1029/2008GL035387.

Hasselmann, K., 1963. A statistical analysis of the generation of microseisms, Rev. Geophys., 1(2), 177-210.

Hillers, G., Graham, N., Campillo, M., Kedar, S., Landès, M. \& Shapiro, N., 2012. Global oceanic microseism sources as seen by seismic arrays and predicted by wave action models, Geochem. Geophys. Geosyst., 13(1), doi:10.1029/2011GC003875.

Köhler, A., Weidle, C. \& Maupin, V., 2012. Crustal and uppermost mantle structure of southern Norway: results from surface wave analysis of ambient seismic noise and earthquake data, Geophys. J. Int., 191(3), $1441-1456$.

Korostelev, F. et al., 2015. Magmatism on rift flanks: Insights from ambient noise phase velocity in Afar region, Geophys. Res. Lett., 42(7), 2179-2188.

Kovach, R.L., 1978. Seismic surface waves and crustal and upper mantle structure, Rev. Geophys., 16(1), 1-13.

Lacoss, R.T., Kelly, E.J. \& Toksöz, M.N., 1969. Estimation of seismic noise structure using arrays, Geophysics, 34(1), 21-38.

Larose, E., Roux, P., Campillo, M. \& Derode, A., 2008. Fluctuations of correlations and Green's function reconstruction: role of scattering, J. Appl. Phys., 103(11), 114907, doi:10.1063/1.2939267.

Lebedev, S., Boonen, J. \& Trampert, J., 2009. Seismic structure of Precambrian lithosphere: new constraints from broad-band surface-wave dispersion, Lithos, 109(1), 96-111.

Lin, F.-C., Moschetti, M.P. \& Ritzwoller, M.H., 2008. Surface wave tomography of the western United States from ambient seismic noise: Rayleigh and Love wave phase velocity maps, Geophys. J. Int., 173(1), 281-298.

Longuet-Higgins, M.S., 1950. A theory of the origin of microseisms, Phil. Trans. R. Soc. A, 243(857), 1-35.

Meier, T., Malischewsky, P. \& Neunhöfer, H., 1997. Reflection and transmission of surface waves at a vertical discontinuity and imaging of lateral heterogeneity using reflected fundamental Rayleigh waves, Bull. seism. Soc. Am., 87(6), 1648-1661.

Meier, T., Dietrich, K., Stöckhert, B. \& Harjes, H.-P., 2004. One-dimensional models of shear wave velocity for the eastern Mediterranean obtained from the inversion of Rayleigh wave phase velocities and tectonic implications, Geophys. J. Int., 156(1), 45-58.

Molinari, I., Raileanu, V. \& Morelli, A., 2012. A crustal model for the Eastern Alps region and a new Moho map in southeastern Europe, Pure appl. Geophys., 169(9), 1575-1588.

Nishida, K., Kawakatsu, H. \& Obara, K., 2008. Three-dimensional crustal S wave velocity structure in Japan using microseismic data recorded by Hinet tiltmeters, J. geophys. Res., 113(B10), doi:10.1029/2007JB005395.

Pedersen, H.A. \& Krüger, F., 2007. Influence of the seismic noise characteristics on noise correlations in the Baltic shield, Geophys. J. Int., 168(1), 197-210.

Prieto, G.A., Lawrence, J.F. \& Beroza, G.C., 2009. Anelastic Earth structure from the coherency of the ambient seismic field, J. geophys. Res., 114(B7), doi:10.1029/2008JB006067.

Ritzwoller, M.H., Lin, F.-C. \& Shen, W., 2011. Ambient noise tomography with a large seismic array, C. R. Geosci., 343(8), 558-570.

Sánchez-Sesma, F.J. \& Campillo, M., 2006. Retrieval of the Green's function from cross correlation: the canonical elastic problem, Bull. seism. Soc. Am., 96(3), 1182-1191.

Sato, Y., 1955. Analysis of dispersed surface waves by means of Fourier Transform: Part 1, Bull. Earthq. Res. Tokyo Univ., 33, 33-47.

Seats, K.J., Lawrence, J.F. \& Prieto, G.A., 2012. Improved ambient noise correlation functions using Welch's method, Geophys. J. Int., 188(2), $513-523$.

Shen, W., Ritzwoller, M.H. \& Schulte-Pelkum, V., 2013. A 3-D model of the crust and uppermost mantle beneath the Central and Western US by joint inversion of receiver functions and surface wave dispersion, J. geophys. Res., 118(1), 262-276.

Snieder, R., 1986. 3-D linearized scattering of surface waves and a formalism for surface wave holography, Geophys. J. Int., 84(3), 581-605. 
Snieder, R., 2004. Extracting the Green's function from the correlation of coda waves: a derivation based on stationary phase, Phys. Rev. E, 69(4), 046610, doi:10.1103/PhysRevE.69.046610.

Soomro, R., Weidle, C., Cristiano, L., Lebedev, S., Meier, T. \& PASSEQ Working Group, 2016. Phase velocities of Rayleigh and Love waves in central and northern Europe from automated, broad-band, interstation measurements, Geophys. J. Int., 204(1), 517-534.

Stehly, L., Campillo, M. \& Shapiro, N., 2006. A study of the seismic noise from its long-range correlation properties, J. geophys. Res., 111(B10), doi:10.1029/2005JB004237.

Stehly, L., Fry, B., Campillo, M., Shapiro, N., Guilbert, J., Boschi, L. \& Giardini, D., 2009. Tomography of the Alpine region from observations of seismic ambient noise, Geophys. J. Int., 178(1), 338-350.

Tsai, V.C., 2009. On establishing the accuracy of noise tomography traveltime measurements in a realistic medium, Geophys. J. Int., 178(3), 15551564.

Tsai, V.C., 2011. Understanding the amplitudes of noise correlation measurements, J. geophys. Res., 116(B9), doi:10.1029/2011JB008483.

Tsai, V.C. \& Moschetti, M.P., 2010. An explicit relationship between timedomain noise correlation and spatial autocorrelation (SPAC) results, Geophys. J. Int., 182(1), 454-460.

Udías, A., 1999. Principles of Seismology, Cambridge Univ. Press.

Verbeke, J., Boschi, L., Stehly, L., Kissling, E. \& Michelini, A., 2012. Highresolution Rayleigh-wave velocity maps of central Europe from a dense ambient-noise data set, Geophys. J. Int., 188(3), 1173-1187.

Weaver, R., Froment, B. \& Campillo, M., 2009. On the correlation of nonisotropically distributed ballistic scalar diffuse waves, J. acoust. Soc. Am., 126(4), 1817-1826.

Weemstra, C., Westra, W., Snieder, R. \& Boschi, L., 2014. On estimating attenuation from the amplitude of the spectrally whitened ambient seismic field, Geophys. J. Int., 197(3), 1770-1788.

Weemstra, C., Snieder, R. \& Boschi, L., 2015. On the estimation of attenuation from the ambient seismic field: inferences from distributions of isotropic point scatterers, Geophys. J. Int., 203(2), 1054-1071.

Wessel, P., Smith, W.H.F., Scharroo, R., Luis, J. \& Wobbe, F., 2013. Generic mapping tools: improved version released, EOS, Trans. Am. geophys. Un., 94(45), 409-410.
Wielandt, E., 1993. Propagation and structural interpretation of non-plane waves, Geophys. J. Int., 113(1), 45-53.

Yang, Y. \& Ritzwoller, M.H., 2008. Characteristics of ambient seismic noise as a source for surface wave tomography, Geochem. Geophys. Geosyst., 9(2), doi:10.1029/2007GC001814.

Yang, Y., Li, A. \& Ritzwoller, M.H., 2008a. Crustal and uppermost mantle structure in southern Africa revealed from ambient noise and teleseismic tomography, Geophys. J. Int., 174(1), 235-248.

Yang, Y., Ritzwoller, M.H., Lin, F.-C., Moschetti, M. \& Shapiro, N.M., 2008b. Structure of the crust and uppermost mantle beneath the western United States revealed by ambient noise and earthquake tomography, $J$. geophys. Res., 113(B12), doi:10.1029/2008JB005833.

Yao, H. \& Van Der Hilst, R.D., 2009. Analysis of ambient noise energy distribution and phase velocity bias in ambient noise tomography, with application to SE Tibet, Geophys. J. Int., 179(2), 11131132.

Yao, H., van Der Hilst, R.D. \& Maarten, V., 2006. Surface-wave array tomography in SE Tibet from ambient seismic noise and two-station analysis-I. Phase velocity maps, Geophys. J. Int., 166(2), 732-744.

Yao, H., Beghein, C. \& Van Der Hilst, R.D., 2008. Surface wave array tomography in SE Tibet from ambient seismic noise and two-station analysisii. Crustal and upper-mantle structure, Geophys. J. Int., 173(1), 205219.

Yao, H., Gouédard, P., Collins, J.A., McGuire, J.J. \& van der Hilst, R.D., 2011. Structure of young East Pacific Rise lithosphere from ambient noise correlation analysis of fundamental-and higher-mode Scholte-Rayleigh waves, C. R. Geosci., 343(8), 571-583.

Yoshizawa, K. \& Ekström, G., 2010. Automated multimode phase speed measurements for high-resolution regional-scale tomography: application to North America, Geophys. J. Int., 183(3), 1538-1558.

Zhou, L., Xie, J., Shen, W., Zheng, Y., Yang, Y., Shi, H. \& Ritzwoller, M.H., 2012. The structure of the crust and uppermost mantle beneath South China from ambient noise and earthquake tomography, Geophys. J. Int., 189(3), 1565-1583.

Zhou, Y., Nolet, G., Dahlen, F. \& Laske, G., 2006. Global upper-mantle structure from finite-frequency surface-wave tomography, J. geophys. Res., 111(B4), doi:10.1029/2005JB003677. 